\title{
Eyes in the sky
}

\section{Interactions between asymptotic giant branch star winds

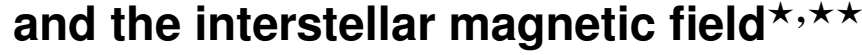

\author{
A. J. van Marle ${ }^{1}$, N. L. J. Cox ${ }^{1}$, and L. Decin ${ }^{1,2}$ \\ 1 KU Leuven, Institute of Astronomy, Celestijnenlaan 200D, 3001 Leuven, Belgium \\ e-mail: AllardJan.vanMarle@ster.kuleuven. be \\ ${ }^{2}$ Universiteit van Amsterdam, Sterrenkundig Instituut Anton Pannekoek, Science Park 904, 1098 Amsterdam, The Netherlands
}

Received 23 June 2014 / Accepted 6 August 2014

\section{ABSTRACT}

\begin{abstract}
Context. The extended circumstellar envelopes (CSEs) of evolved low-mass stars display a large variety of morphologies. Understanding the various mechanisms that give rise to these extended structures is important to trace their mass-loss history. Aims. Here, we aim to examine the role of the interstellar magnetic field in shaping the extended morphologies of slow dusty winds of asymptotic giant branch (AGB) stars in an effort to pin-point the origin of so-called eye shaped CSEs of three carbon-rich AGB stars. In addition, we seek to understand if this pre-planetary nebula (PN) shaping can be responsible for asymmetries observed in PNe. Methods. Hydrodynamical simulations are used to study the effect of typical interstellar magnetic fields on the free-expanding spherical stellar winds as they sweep up the local interstellar medium (ISM).

Results. The simulations show that typical Galactic interstellar magnetic fields of 5 to $10 \mu \mathrm{G}$ are sufficient to alter the spherical expanding shells of AGB stars to appear as the characteristic eye shape revealed by far-infrared observations. The typical sizes of the simulated eyes are in accordance with the observed physical sizes. However, the eye shapes are transient in nature. Depending on the stellar and interstellar conditions, they develop after 20000 to 200000 yrs and last for about 50000 to 500000 yrs, assuming that the star is at rest relative to the local interstellar medium. Once formed, the eye shape develops lateral outflows parallel to the magnetic field. The explosion of a PN in the centre of the eye-shaped dust shell gives rise to an asymmetrical nebula with prominent inward pointing Rayleigh-Taylor instabilities.

Conclusions. Interstellar magnetic fields can clearly affect the shaping of wind-ISM interaction shells. The occurrence of the eyes is most strongly influenced by stellar space motion and ISM density. Observability of this transient phase is favoured for lines-of-sight perpendicular to the interstellar magnetic field direction. The simulations indicate that shaping of the pre-PN envelope can strongly affect the shape and size of PNe.
\end{abstract}

Key words. magnetohydrodynamics (MHD) - circumstellar matter - stars: AGB and post-AGB - ISM: bubbles - ISM: structure ISM: magnetic fields

\section{Introduction}

Asymptotic giant branch (AGB) stars occur in the late stages of the evolution of low mass stars (Habing \& Olofsson 2003; Herwig 2005, and references therein). AGB stars have a high luminosity $\left(\sim 1000-10000 L_{\odot}\right)$, low surface temperature ( 2000-3500 K) (Habing \& Olofsson 2003; Herwig 2005, and references therein) and a slow, dust-driven wind (e.g. Kwok 1975; Lamers \& Cassinelli 1999; Elitzur \& Ivezić 2001). Owing to their high mass loss rate $\left(10^{-7}-10^{-5} M_{\odot} \mathrm{yr}^{-1}\right)$ and the high dust content of their winds (0.1-1\% according to Arndt et al. 1997; Marengo et al. 1997; Hoogzaad et al. 2002; Woods et al. 2012), The AGB stars are considered the primary source of dust in the present-day interstellar medium (ISM) (Habing \& Olofsson 2003; Herwig 2005; Andersen 2007; Zhukovska et al. 2008; Zhukovska \& Henning 2013, and references therein). Because low mass stars do not have a strong wind until they

\footnotetext{
* Herschel is an ESA space observatory with science instruments provided by European-led Principal Investigator consortia and with important participation from NASA.

$\star \star$ Movies are available in electronic form at http://www . aanda.org
}

reach the AGB phase, the AGB wind interacts directly with the ISM, rather than with the stellar wind remnants from earlier evolutionary stages, as would be the case for more massive stars (Villaver et al. 2002, 2012). The collision between the stellar wind and the ISM results in the formation of a high density shell of swept-up interstellar gas, which is pushed outwards into the ISM.

Herschel/PACS (Pilbratt et al. 2010; Poglitsch et al. 2010) observations obtained within the context of the MESS programme (Groenewegen et al. 2011) revealed a large variety of faint and complex extended emission structures around AGB stars and red supergiants (Cox et al. 2012). Several types of morphological classes could be discerned: 1) fermata (bow waves); 2) eyes; 3) rings; 4) irregular sources; and 5) point sources. Fermata-type structures are due to interaction of the slow dusty stellar wind of an evolved star with its surrounding medium. Hydrodynamical simulations of these interactions have been able to predict and reproduce the observations in great detail (e.g. Villaver et al. 2003; Wareing et al. 2007; van Marle et al. 2011; Cox et al. 2012; Decin et al. 2012; van Marle et al. 2014). Ring or shell-type structures can be formed by the interaction of a spherically symmetric wind with a smooth ISM and 

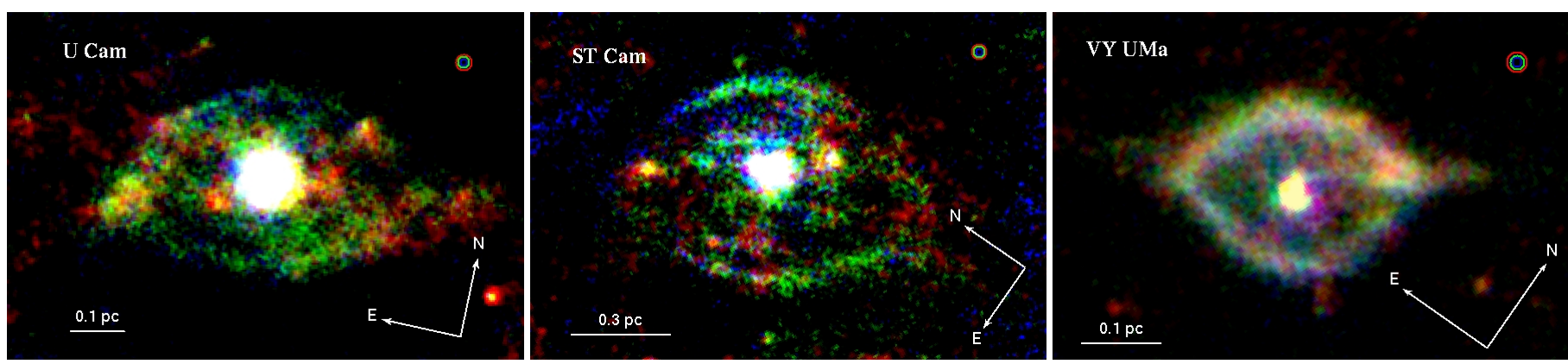

Fig. 1. Herschel/PACS $70 \mu \mathrm{m}, 100 \mu \mathrm{m}$, and $160 \mu \mathrm{m}$ false-colour composite image of U Cam (left), ST Cam (middle), and VY Uma (right). Spatial scales are indicated with the horizontal bar. The compass is oriented with galactic coordinates and the arms are $1^{\prime}$ in length (we note that the images have been rotated to align the symmetry axis for each object). The PACS beam sizes (FWHM) at $70 \mu \mathrm{m}, 100 \mu \mathrm{m}$, and $160 \mu \mathrm{m}$, are represented by the small blue, green, and red circles in the upper right corner of each panel.

Table 1. Basic stellar parameters of the three carbon-rich eye objects.

\begin{tabular}{lccccccccc}
\hline \hline Object & $\begin{array}{c}\text { Distance }^{a} \\
(\mathrm{pc})\end{array}$ & $\begin{array}{c}l \\
\left({ }^{\circ}\right)\end{array}$ & $\begin{array}{c}b \\
\left({ }^{\circ}\right)\end{array}$ & $\begin{array}{c}\dot{M}^{a} \\
M_{\odot} \mathrm{yr}^{-1}\end{array}$ & $\begin{array}{c}v_{\infty}{ }^{a} \\
\left(\mathrm{~km} \mathrm{~s}^{-1}\right)\end{array}$ & $\begin{array}{c}v_{\star}{ }^{b} \\
\left(\mathrm{~km} \mathrm{~s}^{-1}\right)\end{array}$ & $\begin{array}{c}\text { North-east (PA) } \\
\left({ }^{\circ}\right)\end{array}$ & \multicolumn{2}{c}{$\begin{array}{c}\text { Length (pc) } \\
\text { Semi-minor } \\
\text { Semi-major }\end{array}$} \\
\hline U Cam & 525 & 141.1499 & +05.9679 & $2.0 \times 10^{-6}$ & 20.6 & 9.8 & 350.4 & 0.16 & 0.28 \\
ST Cam & 800 & 142.3898 & +14.9236 & $1.1 \times 10^{-6}$ & 9.0 & 28.7 & 247.8 & 0.30 & 0.47 \\
VY UMa & 445 & 139.5898 & +45.4137 & $1.4 \times 10^{-7}$ & 6.0 & 28.2 & 70.5 & 0.10 & 0.16 \\
\hline
\end{tabular}

Notes. ${ }^{(a)}$ Distance, $\dot{M}$, and $v_{\infty}$ adopted from Bergeat \& Chevallier (2005). ${ }^{(b)}$ Space velocities have been corrected for the motion of the sun $[(U$, $\left.V, W)=(11.10,12.24,7.25) \mathrm{km} \mathrm{s}^{-1}\right]$ (Schönrich et al. 2010). ${ }^{(c)}$ Minor and major axis of the eye structures.

are also expected from sudden changes in the mass-loss rate during the AGB phase (e.g. Villaver et al. 2002, 2003; Kerschbaum et al. 2010), which are created by, for example, a thermal pulse (Maercker et al. 2010). Irregular structures of several objects in the MESS sample have also been studied in detail. In these cases, the irregularity was seen to arise from additional mechanisms, such as jets (R Aqr) and spiral structures (W Aql) from a binary interaction (Mayer et al. 2013). Objects classified as a point source were on average more distant, and the absence of extended emission could, hence, represent the angular resolution and surface brightness limits.

\subsection{Eyes in the sky}

The more mysterious class constitutes several carbon-rich evolved stars whose resolved, detached emission is axisymmetric (rather than spherical symmetric) with an eye-like shape. The typical distance from the pupil to the eyelid is $\sim 0.1-0.2 \mathrm{pc}$ with a minor/major axis ratio of $\sim 1.6$. Deeper follow-up PACS 100 and $160 \mu \mathrm{m}$ observations of the original data presented in Cox et al. (2012) confirm the eye shape for three stars, STCam, UCam, and VY UMa ${ }^{1}$. The false-colour images composed of PACS $70 \mu \mathrm{m}, 100 \mu \mathrm{m}$, and $160 \mu \mathrm{m}$ images are shown in Fig. 1. UXDra, previously included in the eye class, could now be re-classified as likely being a ring-type (in this case a detached shell), while the extended emission for AQ Sqr is now clearly of the fermata-type. The classification of the remaining eye objects ( $\mathrm{R} \mathrm{Crt}, \mathrm{V}$ Pav) could not be confirmed and remains uncertain.

\footnotetext{
1 Herschel obsids: 1342242567, 1342242568 ( ST Cam), 1342242577 , 1342242578 (U Cam), 1342243219, 1342243220 (VY UMa), 1342241436, 1342241437 (AQ Sqr), 1342237178, 1342237179 (UX Dra).
}

Basic stellar parameters for the three carbon-rich AGB stars with eye-like extended emission, UCam, STCam, and VY UMa are provided in Table 1. Terminal wind velocities and absolute space motion are accurate to within a few $\mathrm{km} \mathrm{s}^{-1}$. Distances and, hence, mass-loss rates are less accurate. Literature values for distances range from 330 to $525 \mathrm{pc}$ for U Cam, from 390 to 800 pc for ST Cam, and from 445 to 673 pc for VY UMa. For consistency, we adopted the values for the distance, mass loss rate $(\dot{M})$, effective temperature $\left(T_{\text {eff }}\right)$, and wind velocity $\left(v_{\infty}\right)$ from a single reference, Bergeat \& Chevallier (2005).

Some planetary nebulae (PNe), such as M 57, NG 6543 and, especially, MyCn18, also exhibit eye-like shapes. The PNe are formed through the interaction between a fast post-AGB type wind and its slower AGB wind predecessor (Kwok et al. 1978) and particular shapes are usually attributed to density variations in the two winds (Kwok 2000, and references therein). However, such an explanation does not work for the circumstellar shells of AGB stars. The AGB wind collides directly with the surrounding ISM. Density variations in the ISM may deform the shell but are unlikely to result in the sort of mirror symmetry, which we observe in the eye-like structures. The influence of interstellar magnetic fields on the shape of planetary nebulae (PNe) was predicted analytically by Heiligman (1980); Soker \& Dgani (1997) and, more recently, Falceta-Gonçalves \& Monteiro (2014) showed numerical models of planetary nebulae and concluded that only strong magnetic fields $(\simeq 500 \mu \mathrm{G})$ could create a bipolar nebulae.

The influence of interstellar magnetic fields on the circumstellar medium was explored for large scale structures (e.g. superbubbles) by Tomisaka $(1990,1992)$ and Ferriere et al. (1991). More recently, van Marle et al. (2014) showed the effect of an interstellar magnetic field on the bow shock of the red supergiant $\alpha$-Orionis. In this paper, we explore the possibility that the peculiar, eye-like shape of the circumstellar shells around the 
AGB stars UCam, ST Cam, and VY UMa, is the result of the interaction between the AGB wind and the interstellar magnetic field.

\subsection{Layout}

In Sects. 2 and 3 we introduce the analytical approximation and numerical method, respectively. Section 4 presents our results for the numerical simulations, which cover a parameter space for different AGB mass loss rates, interstellar densities, and magnetic field strengths. The validity of our 2.5D approach is confirmed with a 3D model simulation (Sect. 4.3). We also demonstrate the effect of a warm ISM on the shape of th circumstellar shell (Sect. 4.4). In Sect. 5, we discuss the (likely) formation of the eye-shape in the context of AGB winds and the interaction with the interstellar medium. Because $\mathrm{PNe}$ are formed inside the AGB-wind bubbles, it is possible that the shape of an AGB-wind bubble influences the evolution of the PN. Therefore, we briefly demonstrate the evolution of a PN inside the bubbles, as predicted by our simulations (Sect. 6).

\section{Analytical approximation}

The influence of the interstellar magnetic field on the shape of circumstellar bubbles was shown in simulations by Tomisaka (1990, 1992) and Ferriere et al. (1991). These models proved that even a weak interstellar field $(\sim 5 \mu \mathrm{G})$ could significantly alter the shape of the circumstellar bubble. Expansion perpendicular to the magnetic field is slowed down by the magnetic pressure, turning the originally spherical bubble into an ovoid shape. The characteristic length scale at which this occurs can be determined by comparing the ram pressure of the stellar wind:

$P_{\text {ram }}=\frac{\dot{M} v_{\infty}}{4 \pi R^{2}}$

with the magnetic field pressure of the interstellar magnetic field

$P_{\mathrm{B}}=\frac{B^{2}}{2 \mu_{0}}$

with $\dot{M}$ as the stellar mass-loss rate, $v_{\infty}$ the stellar wind velocity, $R$ the distance from the star, $B$ the absolute magnetic field strength, and $\mu_{0}$ the magnetic permeability of the interstellar medium, which is $4 \pi$ in cgs units. Equalizing these two pressure terms gives us,

$R_{\mathrm{B}}=\frac{1}{B} \sqrt{2 \dot{M} v_{\infty}}$

with $R_{\mathrm{B}}$ as the distance from the star at which the ram pressure of the wind becomes smaller than the interstellar magnetic field pressure. If the free-streaming wind expands to this distance, the magnetic field blocks any further expansion of the wind in the direction perpendicular to the field. Therefore, comparing $R_{\mathrm{B}}$ gives us a measure for the size that a circumstellar bubble has to reach in order for the interstellar magnetic field to be come relevant.

Using typical input parameters, we find that $R_{\mathrm{B}}$ is approximately $100 \mathrm{AU}$ for a solar type $\operatorname{star}\left(\dot{M} \simeq 10^{-14} M_{\odot} \mathrm{yr}^{-1}\right.$, $v_{\infty} \simeq 500 \mathrm{~km} \mathrm{~s}^{-1}$, see Fig. 2), which is actually typical for the size of the heliosphere. For a massive star, O-type $\operatorname{star}(\dot{M} \simeq$ $\left.10^{-7} M_{\odot} \mathrm{yr}^{-1}, v_{\infty} \simeq 2000 \mathrm{~km} \mathrm{~s}^{-1}\right), R_{\mathrm{B}}$ is of the order of $2-3 \mathrm{pc}$. For an AGB wind, which has a low velocity $\left(\simeq 10-15 \mathrm{~km} \mathrm{~s}^{-1}\right)$

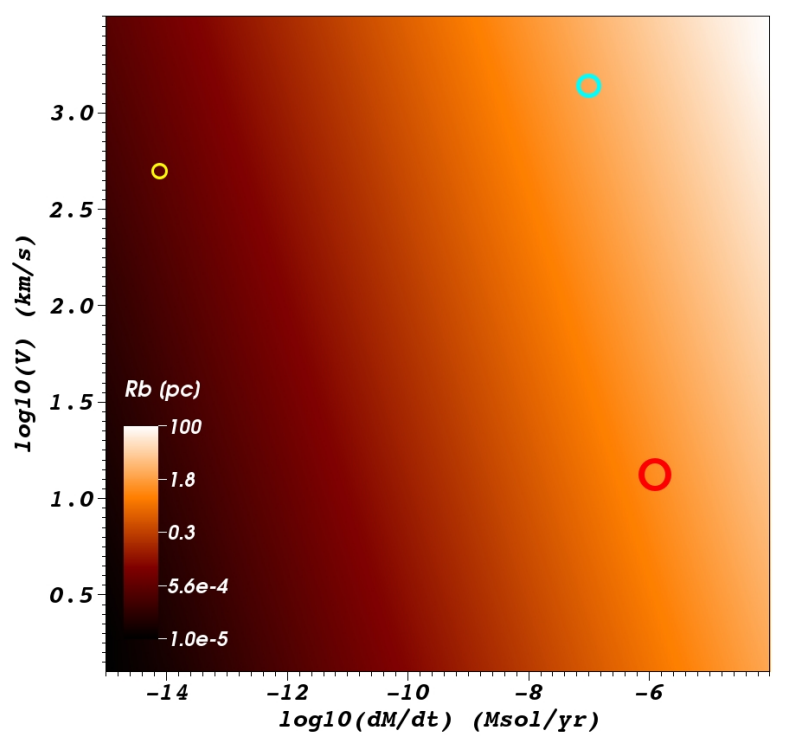

Fig. 2. Magnetic-ram pressure stand-off distance for the wind termination shock as a function of mass loss rate and wind velocity, assuming an interstellar magnetic field strength of $10 \mu \mathrm{G}$. The three coloured circles give the location for a solar type star (yellow), an AGB star (red), and en O-star (blue).

and a relatively high mass loss rate $\left(10^{-7}-10^{-5} M_{\odot} \mathrm{yr}^{-1}\right)$, the stagnation point typically lies at $0.5-1.5 \mathrm{pc}$ (see Fig. 2). Assuming a wind velocity of $10 \mathrm{~km} \mathrm{~s}^{-1}$, a typical AGB lifetime of $1 \mathrm{Myr}$, and no other obstruction to the expanding wind, the AGB wind would reach a maximum distance of $10 \mathrm{pc}$ from the star. Therefore, the interstellar magnetic field cannot be disregarded in models of the circumstellar bubbles of AGB stars. N.B.: we note that $R_{\mathrm{B}}$ denotes the termination shock radius for which further expansion perpendicular to the field stops completely. Even before the wind reaches this point, the influence of the magnetic field starts to deform the spherical symmetry of the circumstellar bubble.

\section{Numerical method}

We use the MPI-AMRVAC hydrodynamics code (Keppens et al. 2012), which solves the conservation equations for mass, momentum, and energy,

$$
\begin{aligned}
\frac{\partial \rho}{\partial t}+\nabla \cdot(\rho \boldsymbol{v}) & =0 \\
\rho\left(\frac{\partial \boldsymbol{v}}{\partial t}+\boldsymbol{v} \cdot \nabla \boldsymbol{v}\right)+\nabla p_{\mathrm{tot}}-\frac{1}{\mu_{0}}(\nabla \times \boldsymbol{B}) \times \boldsymbol{B} & =0 \\
\frac{\partial e}{\partial t}+\nabla \cdot(e \boldsymbol{v})+\nabla \cdot(p \boldsymbol{v})+\left(\frac{\rho}{m_{h}}\right)^{2} \Lambda(T) & =0,
\end{aligned}
$$

with $\rho$ as the density, $\boldsymbol{v}$ the velocity vector, $p_{\text {tot }}$ the sum of the thermal pressure and the magnetic pressure, $\boldsymbol{B}$ the magnetic field, and $m_{\mathrm{h}}$ the hydrogen mass. The energy density $e$ is defined as the sum of thermal, kinetic, and magnetic energy density,

$e=\frac{p}{\gamma-1}+\frac{\rho v^{2}}{2}+\frac{B^{2}}{2 \mu_{0}}$

with the adiabatic index $\gamma=5 / 3$ and $p$ the thermal pressure, and $v=|\boldsymbol{v}|$ and $B=|\boldsymbol{B}|$ as the scale values of the velocity and the magnetic field respectively. We assume ideal magnetohydrodynamics, which means that we have no local source terms for the 
magnetic field and do not account for plasma-related physics. Therefore,

$$
\frac{\partial \boldsymbol{B}}{\partial t}-\nabla \times(\boldsymbol{v} \times \boldsymbol{B})=0 .
$$

We keep the magnetic field divergence-free, using the method described by Powell et al. (1999). The energy equation includes the effect of radiative cooling, which depends on local density, and a temperature-dependent cooling curve for a solar-metallicity gas, $\Lambda(T)$. We obtain $\Lambda(T)$ from Schure et al. (2009), which is a combination of a cooling curve generated with the SPEX code (Kaastra \& Mewe 2000) for temperatures above $10^{4} \mathrm{~K}$ and the cooling curve from Dalgarno \& McCray (1972) for lower temperatures. Schure et al. (2009) provide several curves for the lower temperature regime, depending on the ionization fraction. For our models, we have chosen to assume that the ionization fraction is $10^{-3}$ below $10000 \mathrm{~K}$, which is reasonable for the typical densities in our simulations (e.g. Shu 1983; Fatuzzo et al. 2006). Under these conditions, we can ignore the effect of ambipolar diffusion, which might cause a drift between the charged and neutral particles in the gas. For example, Caselli et al. (1998) found typical ambipolar diffusion time scales of $5 \mathrm{Myr}$ even for the much lower ionization fractions inside dense cores of molecular clouds, which is longer than the expected lifetime of an AGB star.

We set a minimum temperature of $100 \mathrm{~K}$ throughout the simulation, which limits the amount of compression due to radiative cooling, preventing numerical problems that can arise because of extreme compression of radiatively cooling gas.

Our physical domain consists of a $2.5 \mathrm{D}$ cylindrical grid in the $r, z$-plane, covering a physical space of 7.5 by $15 \mathrm{pc}$. At the lowest level of resolution, we have $160 \times 320$ grid points. The grid is allowed to be refined an additional four times, doubling the resolution at each new level of refinement for an effective resolution of $2560 \times 5120$ grid points. This grid is filled with a constant density ISM with a temperature of $100 \mathrm{~K}$ and a magnetic field that runs parallel with the $z$-axis. On the polar axis, we fill a small half-sphere $(R=0.05 \mathrm{pc})$ with stellar wind material. The part of the grid containing this half-sphere is always kept at the highest resolution. We assume the boundaries of the grid to be continuous, so that any signal that reaches the boundary flows out of the grid. The only exception is the boundary condition at the polar axis $(R=0)$, which we assume to be reflective.

\subsection{Input parameters}

The input parameters are specified in Table 2. Simulations A1 and A2 are the control models, which lack an interstellar magnetic field. Simulations B1 through B4 show the interaction of the $\mathrm{AGB}$ wind with a $5 \mu \mathrm{G}$ magnetic field with varying interstellar densities and mass loss rates. Simulations C1 through C4 repeat this for a stronger, $10 \mu \mathrm{G}$, magnetic field. Each simulation runs for $1 \mathrm{Myr}$, which is typical of the timescales of an AGB-star lifetime (Habing \& Olofsson 2003; Herwig 2005, and references therein). For simulation D1, we use the same input parameters as B1 in a low-resolution, 3D model for a period of 500000 years. Finally, for simulation E1, we use the same input parameters as B1 but with a warm ISM set at $8000 \mathrm{~K}$.

\section{Results}

\subsection{Wind expansion without a magnetic field}

With no magnetic field to constrain its expansion (see Fig. 3), the wind-blown bubble is spherical and qualitatively follows
Table 2. Model input parameters.

\begin{tabular}{lccccc}
\hline \hline Simulation & $\begin{array}{c}B_{\mathrm{ISM}} \\
{[\mu \mathrm{G}]}\end{array}$ & $\begin{array}{c}n_{\mathrm{ISM}} \\
{\left[\mathrm{cm}^{-3}\right]}\end{array}$ & $\begin{array}{c}\dot{M} \\
{\left[M_{\odot} \mathrm{yr}^{-1}\right]}\end{array}$ & $\begin{array}{c}v_{\infty} \\
{\left[\mathrm{km} \mathrm{s}^{-1}\right]}\end{array}$ & Grid \\
\hline A1 & 0 & 2 & $10^{-7}$ & 10 & $2.5 \mathrm{D}$ \\
$\mathrm{A} 2$ & 0 & 20 & $10^{-6}$ & 10 & $2.5 \mathrm{D}$ \\
\hline B1 & 5 & 2 & $10^{-7}$ & 10 & $2.5 \mathrm{D}$ \\
$\mathrm{B} 2$ & 5 & 2 & $10^{-6}$ & 10 & $2.5 \mathrm{D}$ \\
$\mathrm{B} 3$ & 5 & 20 & $10^{-7}$ & 10 & $2.5 \mathrm{D}$ \\
$\mathrm{B} 4$ & 5 & 20 & $10^{-6}$ & 10 & $2.5 \mathrm{D}$ \\
\hline $\mathrm{C} 1$ & 10 & 2 & $10^{-7}$ & 10 & $2.5 \mathrm{D}$ \\
$\mathrm{C} 2$ & 10 & 2 & $10^{-6}$ & 10 & $2.5 \mathrm{D}$ \\
$\mathrm{C} 3$ & 10 & 20 & $10^{-7}$ & 10 & $2.5 \mathrm{D}$ \\
$\mathrm{C} 4$ & 10 & 20 & $10^{-6}$ & 10 & $2.5 \mathrm{D}$ \\
\hline D1 & 5 & 2 & $10^{-7}$ & 10 & 3D, \\
& & & & & low resolution \\
\hline E1 & 5 & 2 & $10^{-7}$ & 10 & 2.5D, \\
& & & & & warm ISM \\
\hline
\end{tabular}

the evolution described analytically by Avedisova (1972) and Weaver et al. (1977). Starting from the star, the wind is initially free-streaming with a constant radial velocity and a density that decreases with the distance squared. The wind then passes through the termination shock where it is thermalized and loses most of its kinetic energy to form a hot, low density bubble that expands outwards due to its own thermal pressure. In doing so, it sweeps up a shell of interstellar matter, which moves supersonically into the ISM. The shocked wind and the dense shell of shocked interstellar material are separated by a contact discontinuity. Quantitatively, the comparison with the analytical model is more difficult. The analytical models assume a purely adiabatic interaction (no radiative cooling included) between a fast $\left(\sim 1000 \mathrm{~km} \mathrm{~s}^{-1}\right)$ wind and the interstellar medium, as can be expected for the main sequence phase of massive stars. Obviously, this is not the case here, as the AGB wind has a wind velocity that is two orders of magnitude smaller and a much higher density. As a result, our hot, shocked wind bubble has a temperature of less than $10000 \mathrm{~K}$, rather than the $10^{6}-10^{8} \mathrm{~K}$ expected for massive star bubbles. Nevertheless, Eq. (21) from Weaver et al. (1977) puts the radius of the outer shell for both simulations at $0.5 \mathrm{pc}$ after $0.1 \mathrm{Myr}$ and $2 \mathrm{pc}$ after $1 \mathrm{Myr}$, which matches the results shown in Fig. 3. The fact that simulation A2 consistently has a slightly smaller bubble than simulation A1, even though they should analytically have the same radius, can be explained by energy loss through radiative cooling, which is more effective at the higher densities in this model.

Initially, both the forward and reverse shock are at least partially radiative, causing a high rate of compression, especially in the shell of swept-up ISM. In the case of simulation A2, which has higher densities, the shell is thin enough to show linear thin-shell instabilities (Vishniac 1983), which are caused by the directional asymmetry between the ram pressure acting on the outer boundary of the shell and the thermal pressure exerted on the inner boundary of the shell by the shocked AGB wind. As the bubble expands, the expansion velocity decreases, reducing the compression of the shell, which becomes thicker. Moreover, the density decreases, which reduces the radiative cooling rate (Stevens et al. 1992). As a result, both the forward and reverse shock become purely adiabatic, and the thin-shell instabilities disappear. 

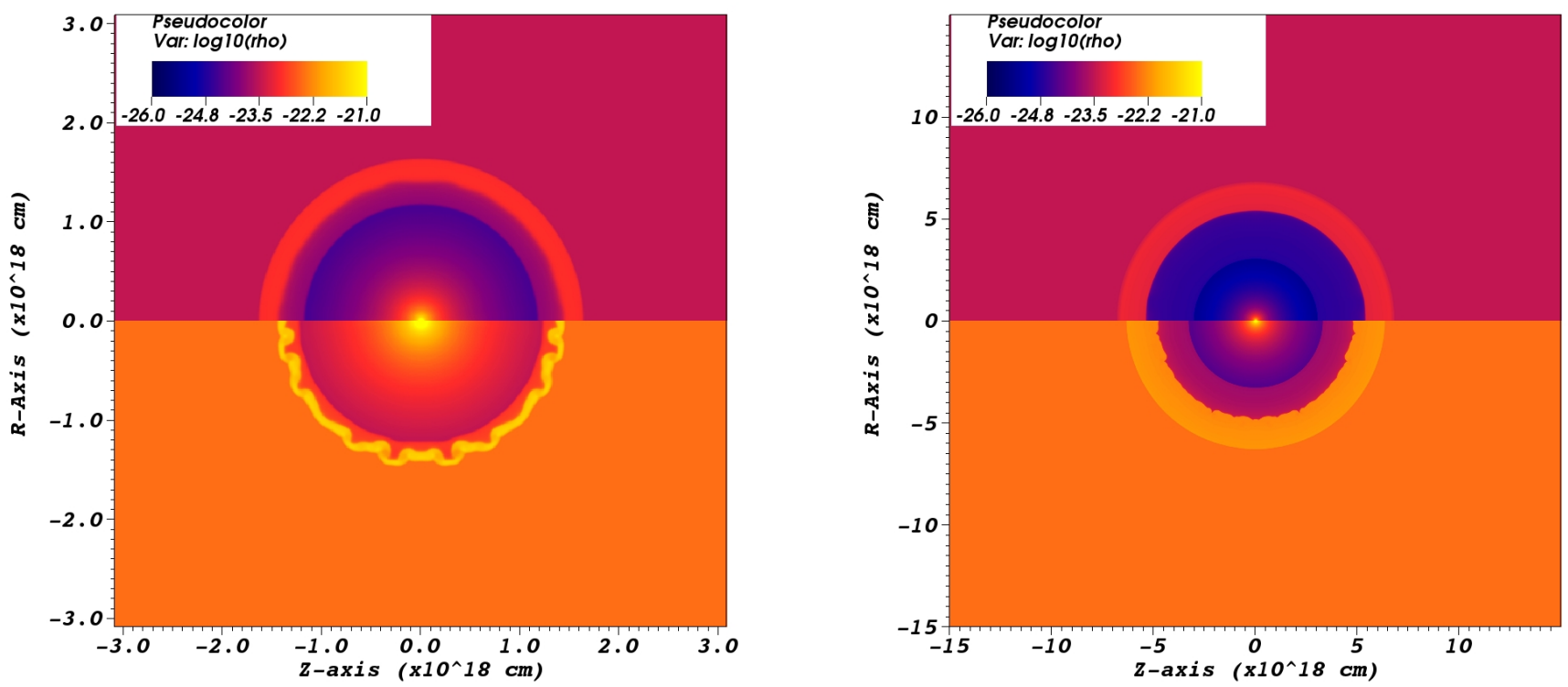

Fig. 3. Gas density of the interstellar medium for simulations A1 (top) and A2 (bottom) after 0.1 (left) and 1 Myr (right). Physical sizes are $2 \times 2$ pc (left panel) and $10 \times 10 \mathrm{pc}$ (right panel). In the earlier stages of the expansion the shells are compressed and simulation A2 shows sign of thin-shell instabilities. Over time the interaction becomes more adiabatic; the compression of the gas diminishes, and the instabilities disappear.
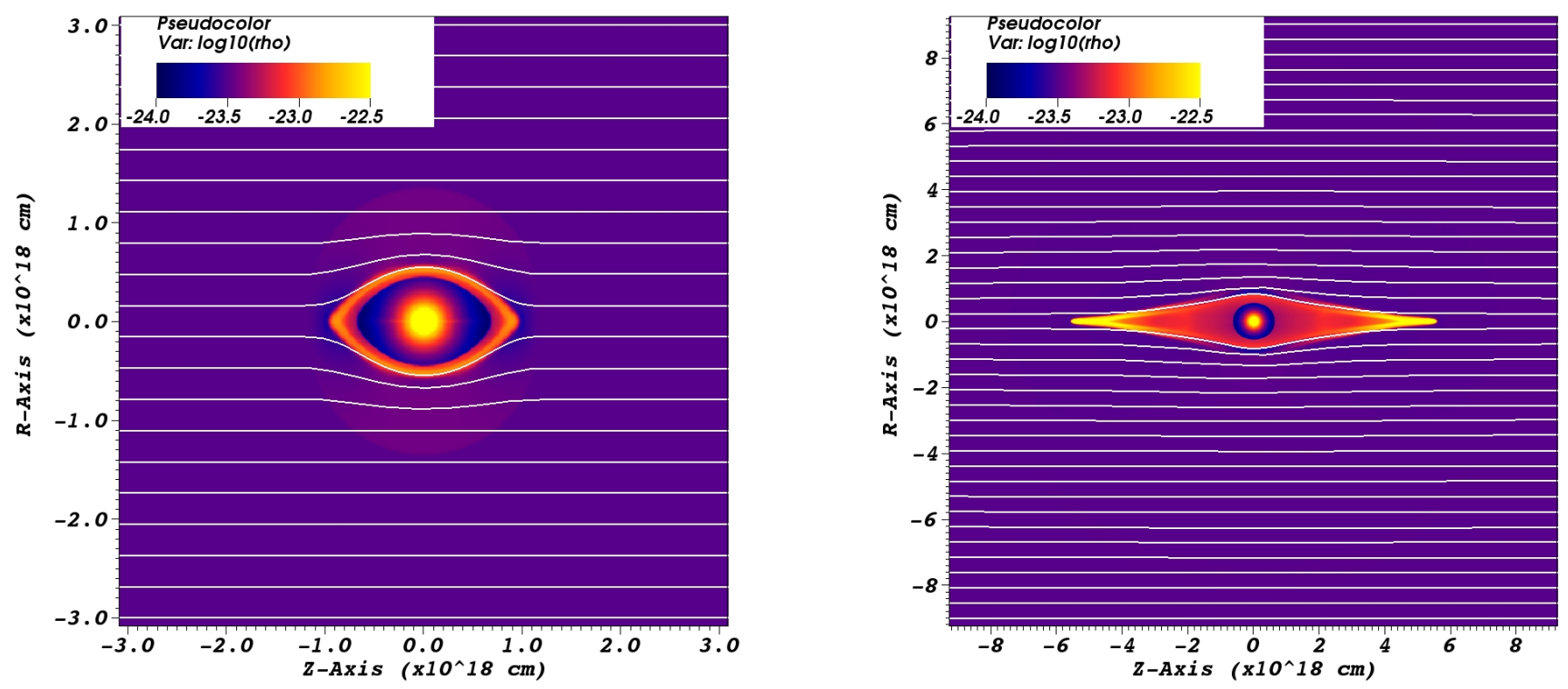

Fig. 4. Gas density and magnetic field lines for simulation B1 after 40000 years (left, the onset of the eye shape) and 300000 years (right, the beginning of the jet formation). Physical scales are $2 \times 2 \mathrm{pc}$ (left panel) and $6 \times 6 \mathrm{pc}$ (right panel).

\subsection{The influence of the interstellar magnetic field}

Even with a weak $(5 \mu \mathrm{G})$ magnetic field (Figs. 4-7), the expansion of the circumstellar bubble quickly becomes a-spherical because the field reduces the expansion in the direction perpendicular to the field. Even before the wind termination shock reaches the critical point $\left(R_{\mathrm{B}}\right)$, where ram pressure and magnetic pressure are in balance, the circumstellar shell is distorted into a shape that starts as ovoid (with the long axis parallel to the magnetic field), which is reminiscent of the results found for super bubbles by Tomisaka $(1990,1992)$ and Ferriere et al. (1991). The magnetic field pressure resists the compression, which confines the shell of shocked ISM in the non-magnetic models. As a result, a relatively small distortion of the interstellar medium density, rather than a highly compressed shell of interstellar matter, moves outward in the direction perpendicular to the field lines. This is most visible in the left panels of Figs. 6 and 7. The interstellar medium is compressed, just ahead of the contact discontinuity, causing a sub-Alfvénic wave to move outwards into the ISM, causing a distortion of the field lines. Behind the contact discontinuity, the shocked wind material, which is stopped from expanding piles up against the ISM. This is fundamentally different from the non-magnetic case, where the dense shell consisted exclusively of interstellar matter. In the magnetic model, the shocked wind itself contributes to the shell, which might lead to a stronger infrared signal because of the high dust-content of the AGB wind. In reality, this distinction would be less clear than in the numerical model, because the dust grains might penetrate from the shocked wind into the shocked ISM because of their large inertia relative to the gas (van Marle et al. 2011). 

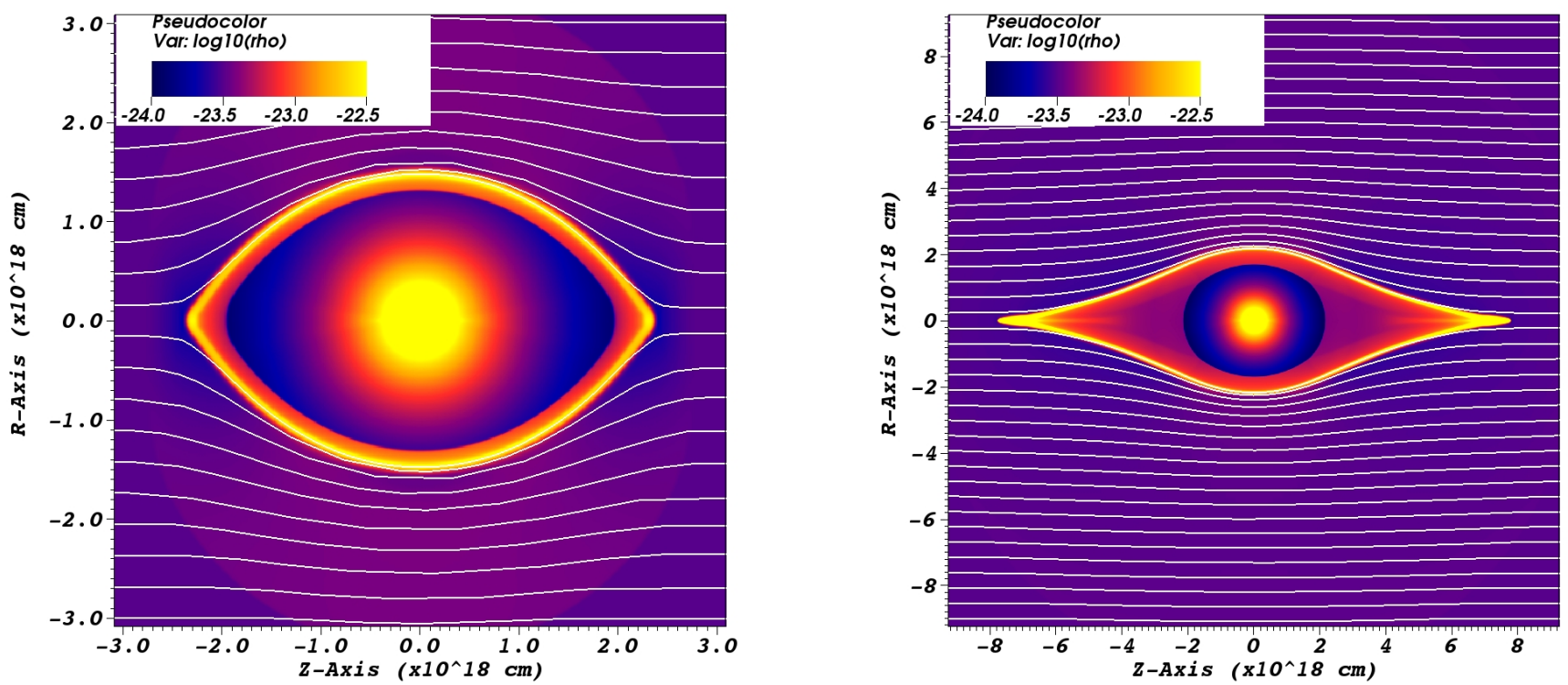

Fig. 5. Similar to Fig. 4 but for simulation B2. The eye shape starts after 100000 years (left), and the collimated flows appear after 380000 years (right). Physical scales are identical to those in Fig. 4.
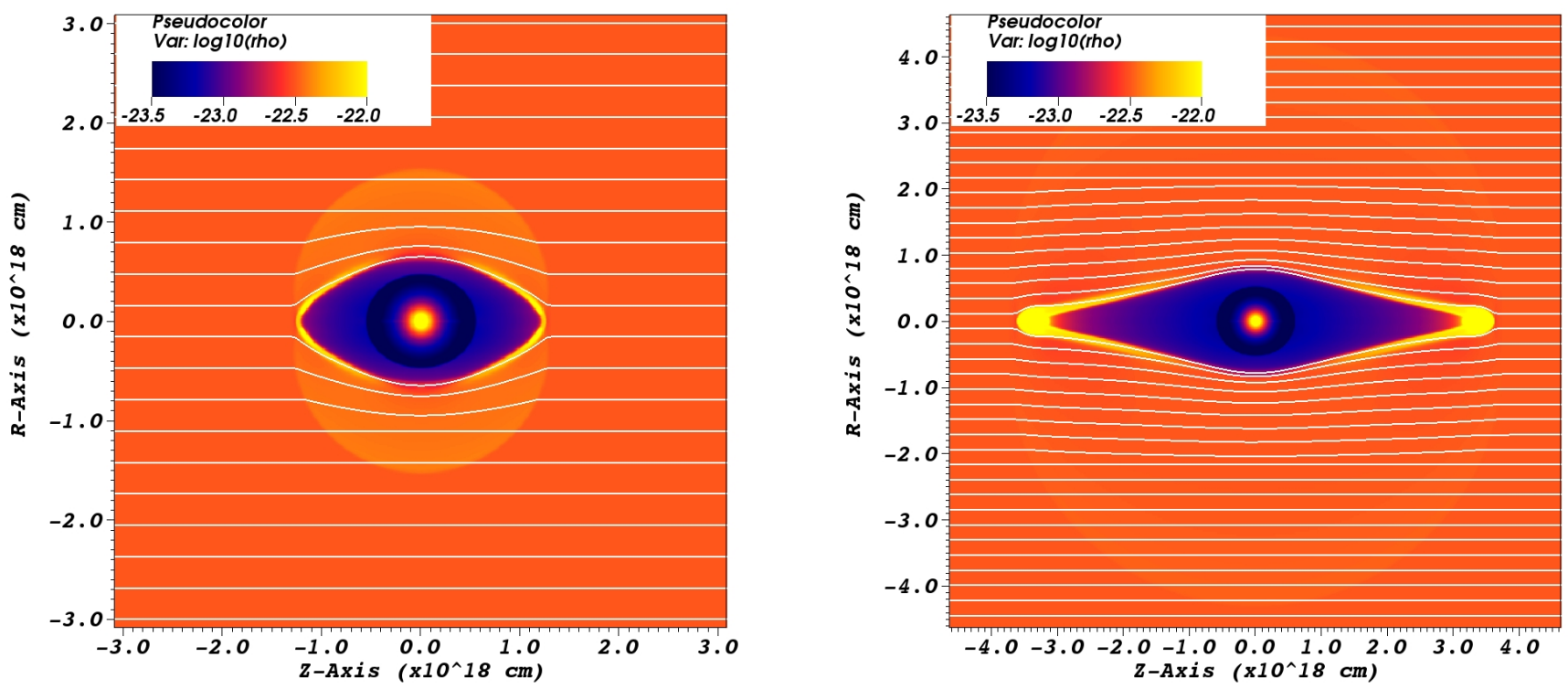

Fig. 6. Similar to Figs. 4, 5 but for simulation B3. The eye shape starts after 120000 years (left) and the collimated flows appear after 430000 years (right). Physical scales are $2 \times 2 \mathrm{pc}$ (left panel) and $3 \times 3 \mathrm{pc}$ (right panel). The scale of this figure deviates from the right panels of Figs. 4,5 , and 7 .

Over time, the asymmetry increases and forms tapering points along the long axis (the $z$-axis in our simulations). This shape, an ovoid with sharp points, eventually turns eye-like as the points becomes elongated. This is caused by the magnetic tension force, which attempts to straighten the field lines by reducing local curvature. The moment this occurs varies from one model to another, depending on the input parameters as demonstrated in Table 3, which shows the time at which the shape becomes eye-like and the diameter along the long axis of the bubble at that moment. Although eye-like is a subjective judgement, we have tried to define a consistent criterion: to be considered eye-like, the shell has to have A) at least 1.5:1 diameter ratio between the long and short axes and B) tapering points. The actual shape of the nebulae as defined in Table 3 for the onset of the eye-like shape is demonstrated in the left panels of
Figs. 4-7, which show the gas density and the shape of the magnetic field-lines.

Eventually, the bubble expands to the point where the magnetic field pressure dominates over the ram pressure of the wind (the termination shock reaches $R_{\mathrm{B}}$ ). Once this occurs, all expansion perpendicular to the field lines ceases. Expansion parallel to the field continues, because the stellar wind continues the feed mass and energy into the bubble. This leads to the formation of elongated structures consisting of collimated outflows on either side of the circumstellar bubble, which extend parallel to the magnetic field lines. Again, the age and largest diameter of the bubble at the start of the collimated flows is noted in Table 3. The density of the circumstellar nebulae and the shape of the interstellar magnetic field lines is shown in the right-side panels of Figs. 4-7. Figure 8 shows the density and temperature of 

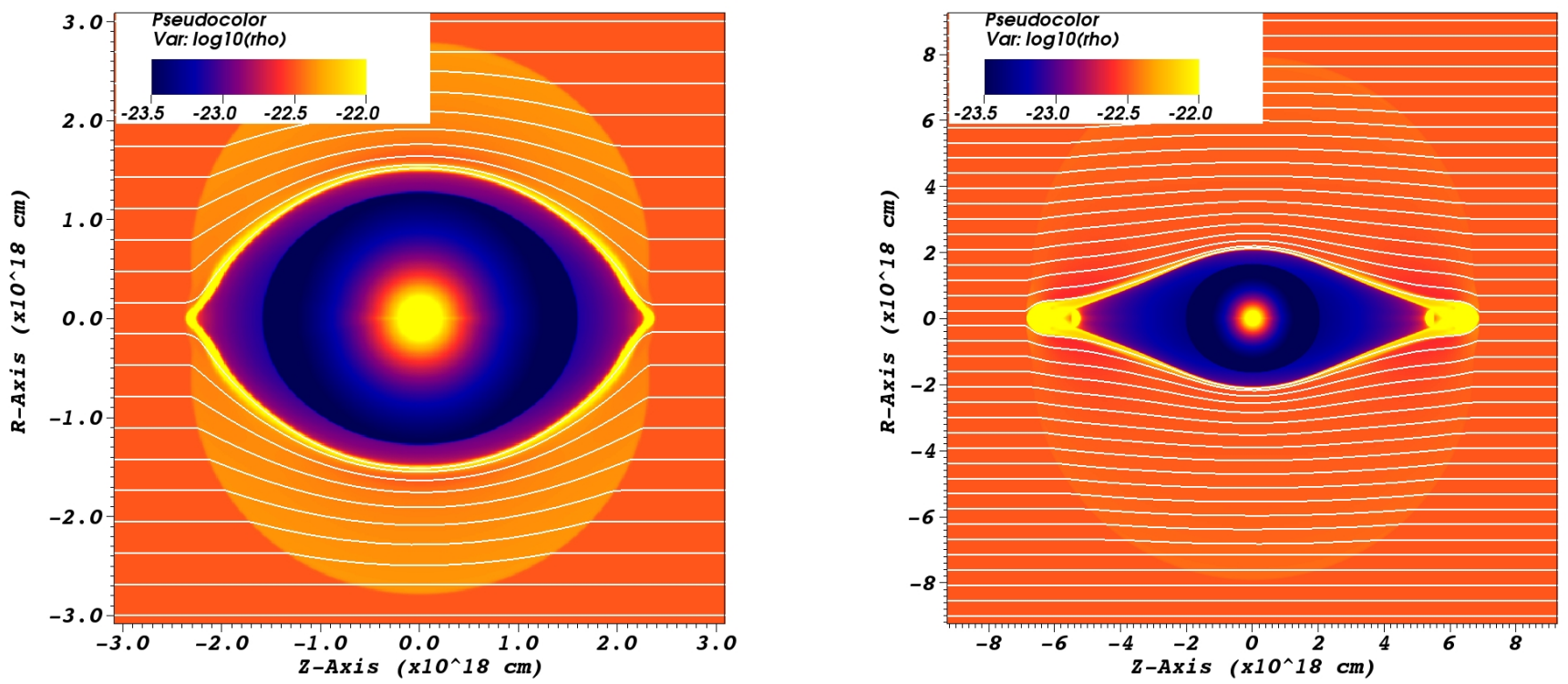

Fig. 7. Similar to Figs. 4-6 but for simulation B4. The eye shape starts after 190000 years (left) and the collimated flows appear after 730000 years (right). Physical scales are identical to those in Figs. 4, 5.

simulation B1 after 1 Myr, which clearly demonstrates the behaviour of the collimated flows, which have stretched to approximately $5 \mathrm{pc}$ on each side of the star at this time.

The simulations with a $10 \mu \mathrm{G}$ interstellar magnetic field (Figs. 9-12) follow the same pattern as for the $5 \mu \mathrm{G}$ field. Because the magnetic field is stronger, it starts to dominate the interaction in an earlier phase of the evolution, reducing the time and length scales for the onset of the eye-like shape (Table 3 and left panels of Figs. 9-12) and the formation of collimated flows (Table 3 and right panels of Figs. 9-12).

When compared to the analytical prediction of the standoff distance between the stellar wind and the magnetic field (Sect. 2), the eye-like structures tend to be smaller, because they start to form in the time when the magnetic field influences the shape of the shell. This, however, does not yet dominate completely over the ram pressure of the wind. The scalingdependence on the magnetic field strength seems to match the analytical approximation. As a pattern, size tends to depend more on mass loss rate than ISM density.

\section{3. $3 D$ vs. $2 D$}

To determine whether the use of a $2.5 \mathrm{D}$, cylindrically-symmetric grid influences the shape of the bubble, we run a single model in $3 \mathrm{D}$, using a Cartesian grid and the same input parameters as for simulation B1. For this 3D model, we reduce the resolution by a factor of four and limit the size of the grid in the directions perpendicular to the field to decrease the computation time. Figure 13 shows cross-sections of the result after 40000 and 300000 years (the onset of the eye-like shape and the start of the collimated flows, respectively.) Because of the lower resolution, the circumstellar shell is not as well resolved, but the result is qualitatively comparable to the $2.5 \mathrm{D}$ model. The nebula initially takes the form of a lemon, which would appear as an eye-like shape (left and centre panels of Fig. 13) when projected on the sky. During the later stages of the evolutsion the 3D model forms the same collimated outflows as found in the $2.5 \mathrm{D}$ simulations (right panel of Fig. 13).

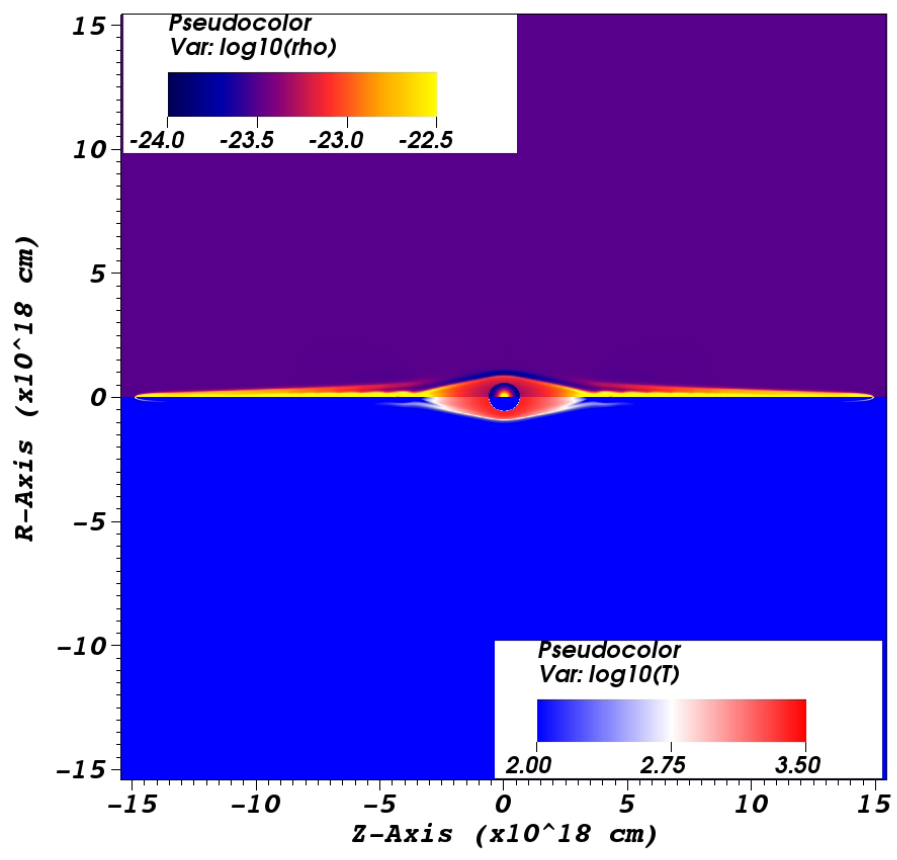

Fig. 8. Density (top) and temperature (bottom) in [cgs.] units for simulation B1 after 1 Myr. The collimated flows have taken on a jet-like appearance and stretch approximately $5 \mathrm{pc}$ in each direction.

\subsection{Warm vs. cold ISM}

Figure 14 shows the result of simulation E1 at the same moments in time as Fig. 4 to demonstrate the effect of a warm ISM $(8000 \mathrm{~K})$. Both the eye-shape (left panel) and the collimated flows (right panel) are clearly visible. However, the nebula is significantly smaller. After 40000 years (left panel), the major axis of the nebula is only $0.45 \mathrm{pc}$ rather than the $0.67 \mathrm{pc}$ that is found for the cold ISM (simulation B1). Similarly, after 300000 years (right panel) the major axis is approximately $2 \mathrm{pc}$, as opposed to $4 \mathrm{pc}$ for simulation B1. This reduction in size is caused by the thermal pressure of the ISM, which constrains the expansion. 

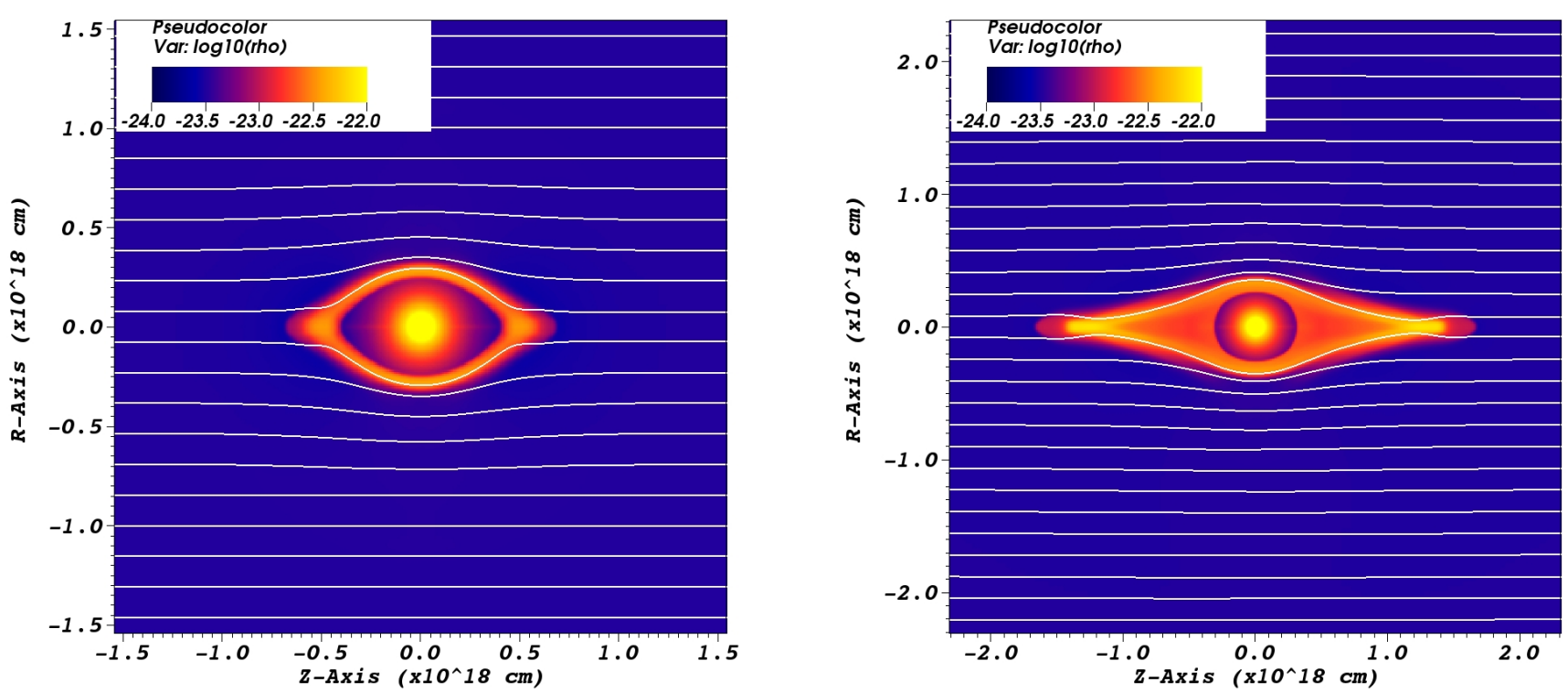

Fig. 9. Gas density and magnetic field lines for simulation $\mathrm{C} 1$ after 20000 years (left, the onset of the eye shape) and 60000 years (right, the beginning of the jet formation.) Physical scales are $1 \times 1 \mathrm{pc}$ (left panel) and $1.5 \times 1.5 \mathrm{pc}$ (right panel).
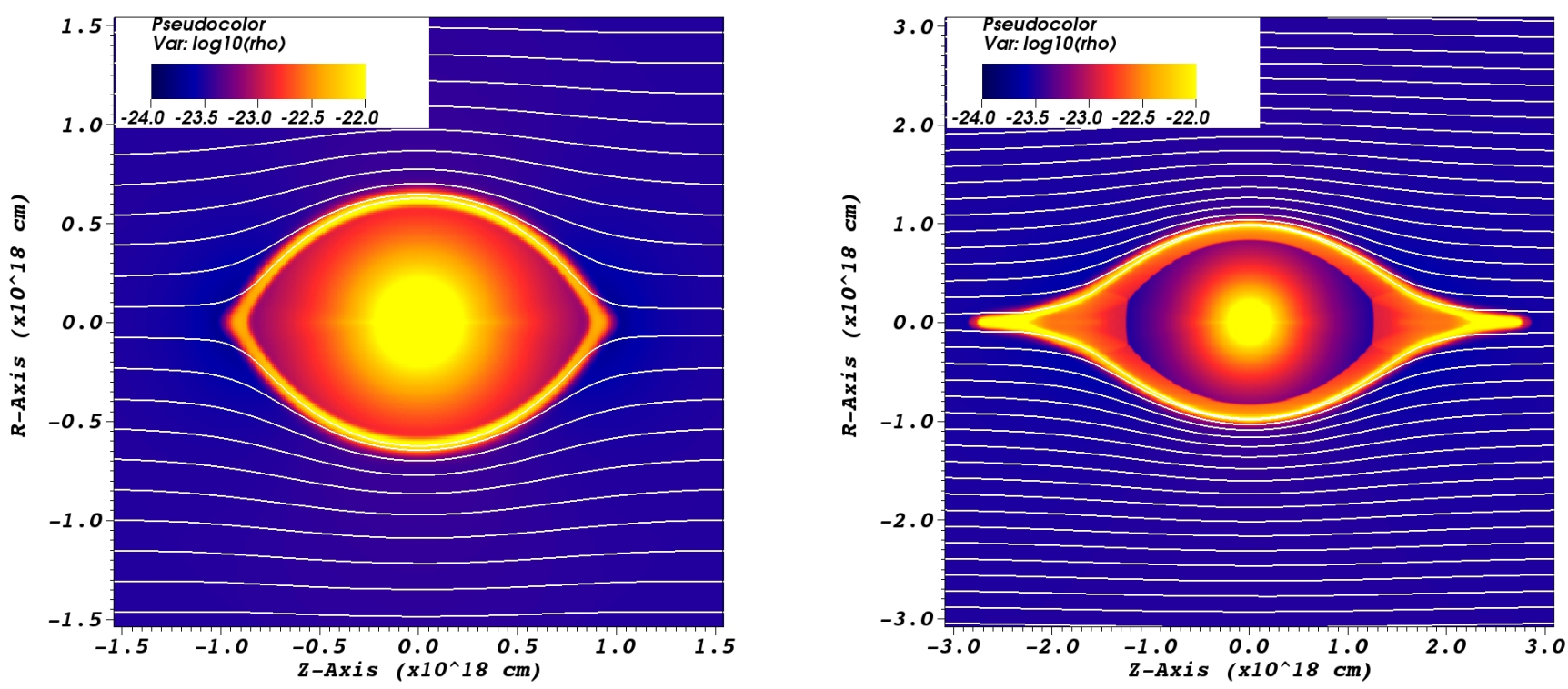

Fig. 10. Similar to Fig. 9 but for simulation C2. The eye shape starts after 30000 years $(l e f t)$ and the collimated flows appear after 110000 years $($ right $)$. Physical scales are $1 \times 1 \mathrm{pc}$ (left panel) and $2 \times 2 \mathrm{pc}$ (right panel). We note the scaling difference between the right panel of this figure and the right panel of Fig. 9.

\section{Discussion}

\subsection{Shape}

The question is not how can we obtain an eye shape, but rather why we do not see more of them? As long as an AGB wind interacts with an interstellar magnetic field, the bubble will sooner, or later appear eye-like. However, for us to observe it, it also has to be visible. Using Tielens (Eq. (5.44) from 2005), we find that carbon-based dust grains has a temperature of approximately $55 \mathrm{~K}$ at a distance of $0.1 \mathrm{pc}$ for a stellar luminosity of $10^{3} L_{\odot}$ and a grain size of $0.1 \mu \mathrm{m}$, whereas the dust temperature at $1.0 \mathrm{pc}$ is reduced to about $25 \mathrm{~K}$, which is approximately the same temperature as the dust in the ISM (Tielens 2005). As a result, the shell may not be visible against the background of the interstellar medium, though the difference between the carbon-rich dust of the AGB wind and the oxygen rich interstellar dust may still be observable. The eye-shaped nebulae in our simulations tend to be larger than the three observed eyes and may be unobservable for part or all of their eye-like phases. Comparing the cross-sections in Table 3 with the semi-major axes listed in Table 1 shows that the simulation results are already as large as, if not larger than, the observed nebulae at the onset of the eye-like phase. The fact that a warm ISM reduces the size of the nebula without compromising the eye-shape, as demonstrated in Fig. 14, may indicate that eye-like nebulae are more likely to be found in regions with a warm ISM.

A second limitation is caused by stellar motion. If the star moves supersonically through the ISM, this eventually 
A. J. van Marle et al.: Eyes in the sky
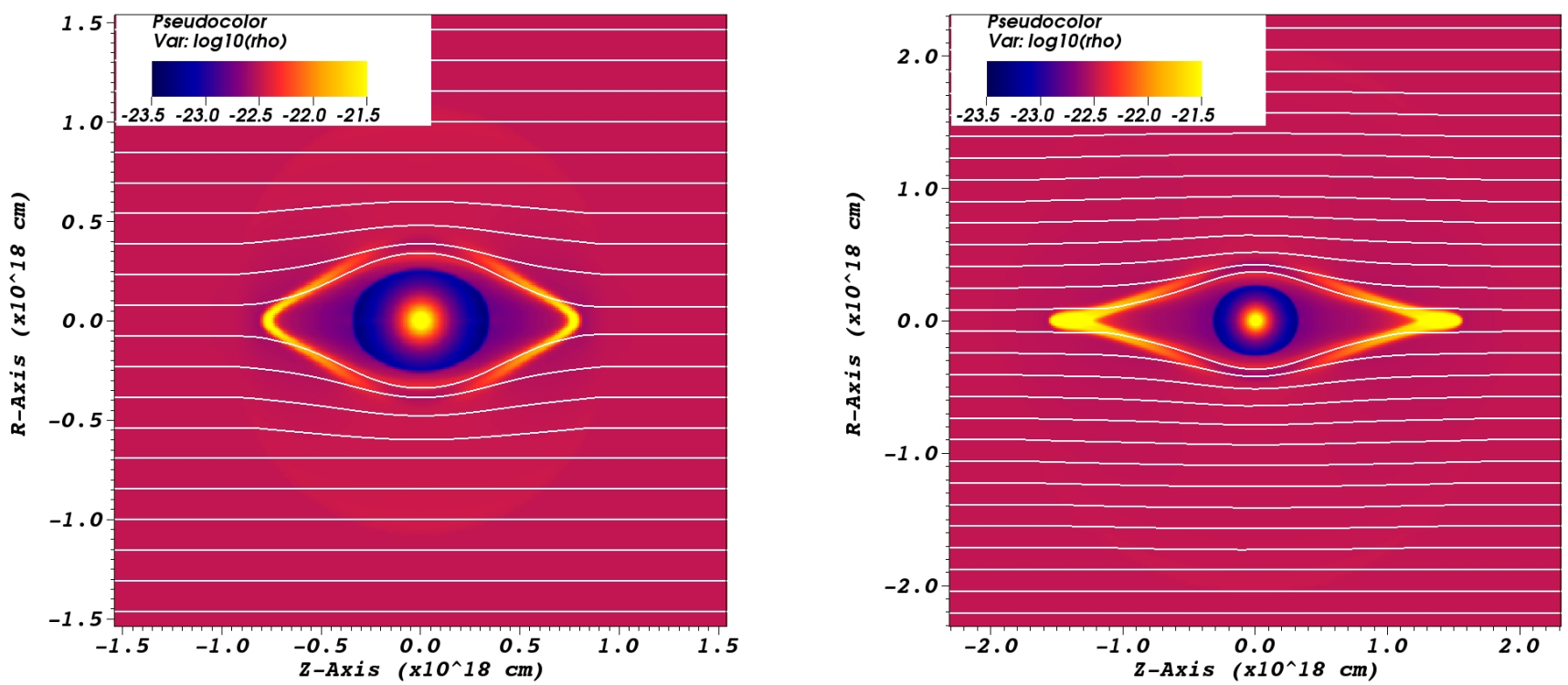

Fig. 11. Similar to Figs. 9, 10, but for simulation C3. The eye shape starts after 50000 years (left), and the collimated flows appear after 110000 years (right). Physical scales identical to those in Fig. 9.
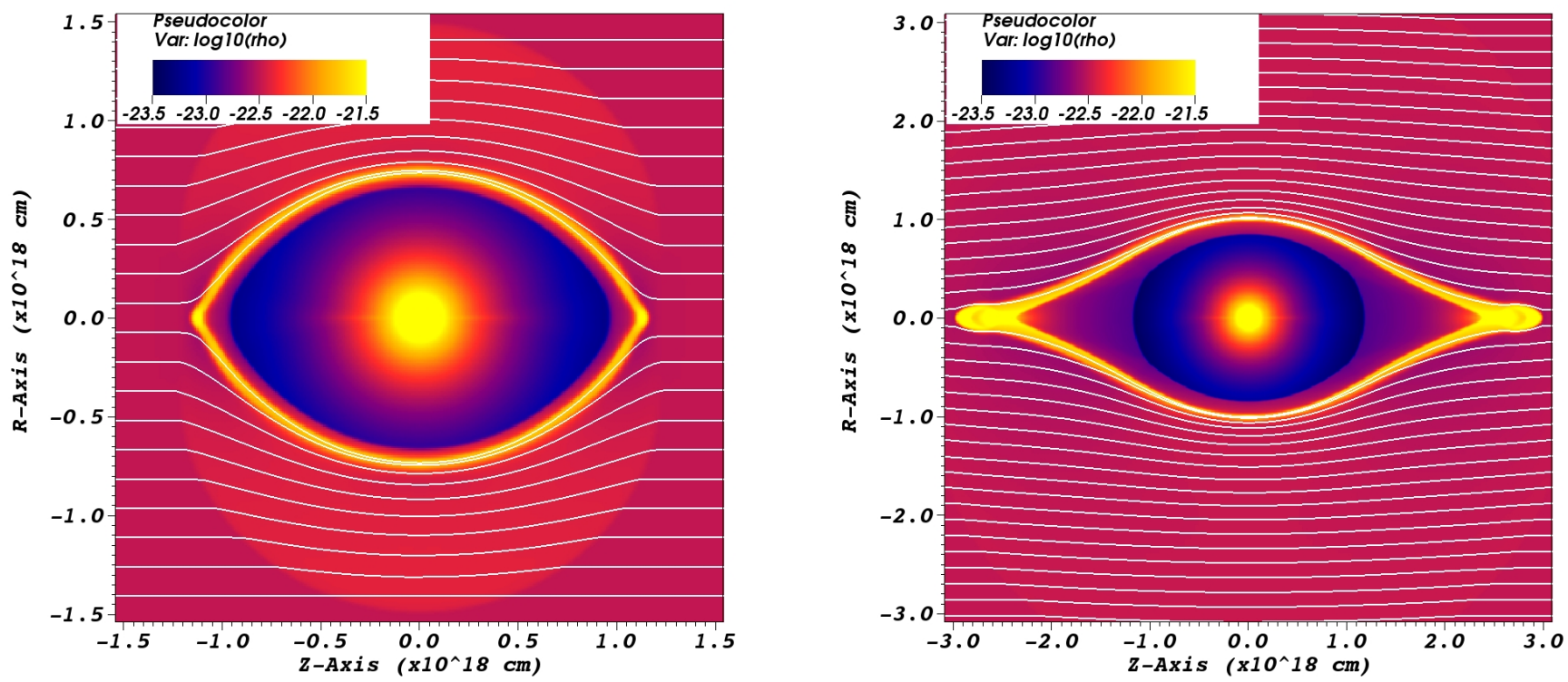

Fig. 12. Similar to Figs. 9-11, but for simulation C4. The eye shape starts after 60000 years (left) and the collimated flows appear after 190000 years (right). Physical scales are identical to those in Fig. 10.

determines the shape of the circumstellar shell, which forms a fermata-type bow shock (Cox et al. 2012). An eye-like shape can only occur, as long as the wind is still expanding in all directions before it reaches a ram pressure balance with the ISM. Assuming the parameters of simulation B1 and a stellar motion of $20 \mathrm{~km} \mathrm{~s}^{-1}$, the stand-off distance of the bow shock $\left(R_{\mathrm{D}}\right)$ would be $0.063 \mathrm{pc}$ (Wilkin 1996), an order of magnitude smaller than the initial eye-like structure (Table 3 ). Therefore, if the star, as modelled in Simulation B1, were to travel at $20 \mathrm{~km} \mathrm{~s}^{-1}$, it would never form an eye-like nebula, but would, almost from the start appear to have a fermata-type nebula. As a result, we can expect eye-like structures to occur primarily around stars in a low density ISM. This delays the formation of a bow shock and allows the star to form a nebula that is initially eye-like before it succumbs to the fermata shape.
Changes in $\dot{M}$ or $v_{\infty}$ have little effect on whether the ram pressure of the ISM or magnetic pressure dominates, since both $R_{\mathrm{D}}$ and $R_{\mathrm{B}}$ scale with the square-root of these quantities. On the other hand, $\mathrm{n}_{\mathrm{ISM}}$ and the spatial velocity $\left(v_{\star}\right)$ are important because they determine $R_{\mathrm{D}}\left(R_{\mathrm{B}}\right.$ being independent of these parameters). An additional limiting factor is $\mathrm{T}_{\text {ISM }}$ because the star needs to move supersonically to give rise to a bow shock. Using the characteristics of the interstellar medium, as defined by Tielens (2005, Chapter 1), typical stellar motion $\left(10-100 \mathrm{~km} \mathrm{~s}^{-1}\right)$ (Binney \& Merrifield 1998) is supersonic in cold $(\lesssim 100 \mathrm{~K})$ or warm $(\simeq 8000 \mathrm{~K})$ ISM but would be subsonic in the hot intercloud medium.

Finally, we have to consider the angle at which we observe the shape. The shell only appears eye-like if our line of sight is approximately perpendicular to magnetic field (cf. Sect. 4.3). 

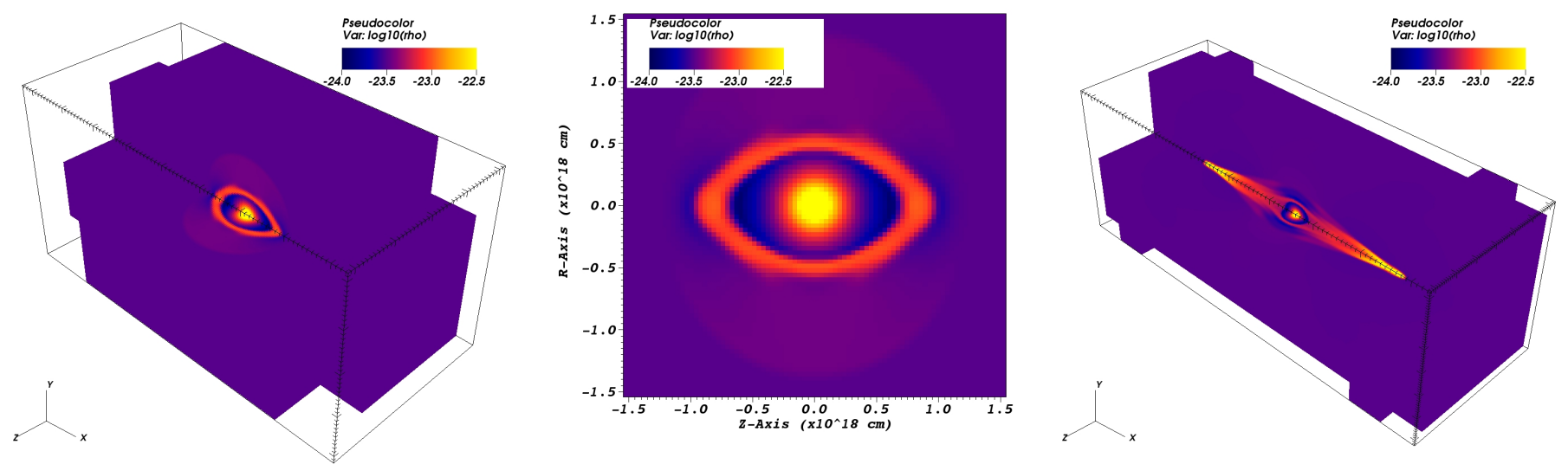

Fig. 13. 3D results for simulation D1, showing the circumstellar density after 40000 (left and centre) and 300000 years (right), respectively. The centre panel shows a slice through the 3D data-cube that can be compared directly to the left panel of Fig. 4. Although the shell is not as well resolved as for the 2.5D models, both the eye-like shape and the collimated flows are clearly visible. This confirms the validity of the $2.5 \mathrm{D}$ models.
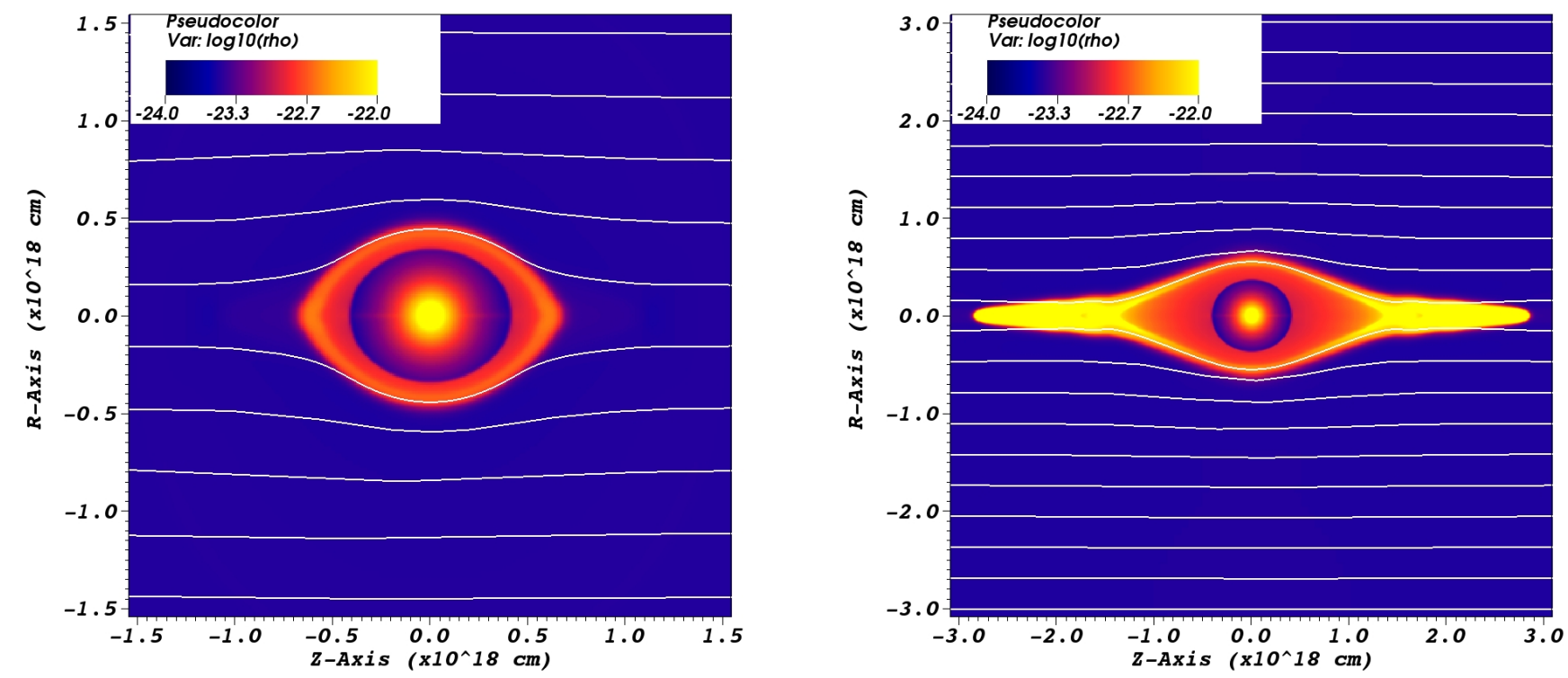

Fig. 14. Similar to Fig. 4 for simulation E1 at the same moments in time. Physical scales are $1 \times 1 \mathrm{pc}($ left panel) and $2 \times 2 \mathrm{pc}($ right panel) and the colour bar has been adjusted to show the density structure. The physical size of the nebula is significantly smaller than for the cold ISM.

When observed along the direction of the magnetic field, the shell appears ring-like. Unfortunately, this cannot be confirmed for the observed eye-shapes due to lack of knowledge of the local magnetic field direction (and strength). The angle of orientation of the eyes with respect to the Galactic plane only provides a rough indication (Fig. 1).

\subsubsection{Ring-like nebulae}

Although the results of simulations $\mathrm{A} 1$ and $\mathrm{A} 2$ reproduce the ring-like nebulae observed with Herschel (Cox et al. 2012), this should not be interpreted as proof that the presence of ring-like nebulae indicates the absence of an interstellar magnetic field. Not only can both eye-like and fermata nebulae appear as rings, depending on the angle of observation, but a ring-like nebula can also be formed through a spherically symmetric outburst caused by a thermal pulse (Villaver et al. 2002, 2003). If such an outburst occurs, the ring-shaped ejecta will initially expand into the free-streaming AGB wind. During this stage, the ring has no interaction with the interstellar medium. This is probably the explanation for the presence of a ring-like structure inside the fermata-type shell observed around R Scl (Cox et al. 2012).

Even when interacting directly with the interstellar magnetic field, the outer shell may appear ring-like as long as the ram pressure of the wind at the termination shock dominates over both the interstellar magnetic pressure and the magnetic tension force. This occurs if either the interstellar magnetic field is weak and/or the shell is still young with the termination shock close to the star.

\subsection{Compression}

Although the non-magnetic simulations (A1 and A2) start out with partially radiative shocks, the shocks over time become adiabatic, and the compression is reduced. All simulations that include magnetic fields show a completely different development: the additional pressure exerted on the shell by the interstellar magnetic field causes the shocked wind to pile up at the contact 
discontinuity. The local density increases, which makes the radiative cooling more efficient. As a result, the reverse shock tends to remain at least partially radiative throughout the evolution of the circumstellar bubble.

\subsection{Gas-dust connection}

The observations done with Herschel (Fig. 1) show the dust rather than the gas. Numerical simulations of the $\alpha$-Orionis bowshock (van Marle et al. 2011; Cox et al. 2012; Decin et al. 2012) show that small dust grains are strongly coupled to the gas and remain within the shocked gas region. They are expected to pile up at the contact discontinuity (van Marle et al. 2011). Therefore, we can expect that such a shape would also be visible in the infrared, as long as the AGB wind, which contains the most dust forms an eye-like shape. These numerical models did not include any interaction between dust grains and a magnetic field. If the dust grains have an electric charge, caused by moving through a plasma and the interaction with the interstellar UV field, they will be tied to the magnetic field, which stops them from moving deep into the ISM. In general, the hydrodynamical interaction in the wind-ISM interaction region are not very energetic, due to the low velocity of the AGB wind. As a result, dust-grains are likely to survive the transition through the shock.

\subsection{Would the outflows be visible?}

Over time, the collimated outflows extend along the field lines (Fig. 8). However, no such structures have been observed, except, possibly, for the case of VY UMa (See Fig. 1), and even there, they have nowhere near the length of the collimated flows found in the simulations. There are several contributing factors that reduce the likelihood of observing these collimated flows:

1. They only occur in the later stages of the nebula evolution. By this time the nebula is often distorted by the stellar motion, which destroys the collimated flows, just as it destroys the eye-shape.

2. The dust in the jets is too cold. The collimated flows only form in the later stages of the evolution of the bubble. By that time, the bubble has expanded, increasing the distance to the central star, which reduces the dust temperature.

3. The flows are kept collimated by the magnetic field tension and pressure. Owing to turbulence, the interstellar magnetic field is unlikely to be completely constant in strength and direction over long distances (Minter \& Spangler 1996; Han et al. 2004), and any variation in the direction of the field would break up the collimated flows.

\section{Influence of interstellar magnetic fields on the evolution of planetary nebulae}

\subsection{Premise}

Planetary nebulae are the product of the interaction between a fast, low density post-AGB wind and its AGB wind predecessor (Kwok et al. 1978). This means that the interstellar magnetic field, which shapes the AGB-wind bubble, can indirectly influence the shape of the PN. However, under most circumstances, this is unlikely. The lifetime of a PN is limited by the evolution of its progenitor star, which typically moves from the initial post-AGB phase to the white dwarf stage within approximately 10000 years (Kwok 2000, and references therein). Once
Table 3. Age and size of eye-shapes.

\begin{tabular}{lcccc}
\hline \hline Simulation & \multicolumn{2}{c}{ Start eye-shape $^{a}$} & \multicolumn{2}{c}{ Start collimation $^{a}$} \\
& {$[\times 1000 \mathrm{yr}]$} & {$[\mathrm{pc}]$} & {$[\times 1000 \mathrm{yr}]$} & {$[\mathrm{pc}]$} \\
\hline B1 & 40 & 0.67 & 300 & 4.0 \\
B2 & 100 & 1.6 & 380 & 5.3 \\
B3 & 120 & 0.83 & 430 & 2.3 \\
B4 & 190 & 1.6 & 730 & 4.7 \\
\hline C1 & 20 & 0.4 & 60 & 1.0 \\
C2 & 30 & 0.65 & 110 & 2.0 \\
C3 & 50 & 0.53 & 110 & 1.0 \\
C4 & 60 & 0.8 & 190 & 2.0 \\
\hline
\end{tabular}

Notes. ${ }^{(a)}$ The criteria used to define the eye-shape and collimated flows are described in Sect. 4.

this happens, the PN, which is no longer driven by a stellar wind or ionized by a strong radiation source, becomes invisible. As our simulations A1 and A2 show, the typical AGB wind bubbles (disregarding the interstellar magnetic field) reach radii of about $2 \mathrm{pc}$ within the lifetime of an AGB star. For PNe to interact with the interstellar medium at all, they would have to reach this distance within the 10000 years of its own lifetime, which requires a minimum velocity of approximately $200 \mathrm{~km} \mathrm{~s}^{-1}$. This is an order of magnitude higher than the typical expansion velocities found for PNe (Terzian 1997).

One exception to this rule is the nearby PN Sh-216, which has an estimated radius of approximately $1.5 \mathrm{pc}$. (Tweedy et al. 1995). The expansion speed of the shell of this particular PN is estimated at $4 \mathrm{~km} \mathrm{~s}^{-1}$, which is less than a typical AGB wind velocity; whereas the shell should actually exceed the speed of the AGB wind that it is sweeping up. This indicates that the shell is interacting directly with the ambient interstellar medium. Ransom et al. (2008) showed polarization data, probing the magnetic field in Sh-216, which indicate that the interstellar magnetic field is being pushed outward by the expanding shell. Similarly, Soker \& Zucker (1997) showed that an interaction may have taken place between the outer halo of NGC 6894 and the interstellar magnetic field.

However, as shown in our simulations, the interstellar magnetic field can reduce the size of the AGB-wind bubble and, if the AGB wind bubble is small enough, the PN starts to interact directly with the ISM, once it has swept-up the AGB wind. For example, for simulation $\mathrm{C} 1$, the analytical value for $R_{\mathrm{B}}$ is only $0.11 \mathrm{pc}$, which is born out by the simulation result (See Fig. 9). For a PN expansion speed of $20 \mathrm{~km} \mathrm{~s}^{-1}$, this would mean that the PN reaches the wind termination shock after only 5500 years, which is well within the lifetime of a typical PN. Falceta-Gonçalves \& Monteiro (2014) showed simulations of $\mathrm{PNe}$ interacting with interstellar magnetic fields and concluded that only very strong fields $(\simeq 500 \mu \mathrm{G})$ could significantly influence the shape of PNe. However, the study by Falceta-Gonçalves \& Monteiro (2014) focused on bipolar PNe. In this work, we simply intend to demonstrate if, and how, the distortion of the AGB-wind bubbles by the interstellar magnetic field can influence the evolution of nebulae created in subsequent evolutionary phases.

As an example, we choose to use simulation $\mathrm{C} 2$, (high mass loss rate, low interstellar density and a $10 \mu \mathrm{G}$ interstellar magnetic field) and use it as a basis for a PN simulation. Using the result of this simulation at $0.1 \mathrm{Myr}$ as basis we launch a fast, post-AGB wind inside the wind blown bubble and follow its evolution over time. This is NOT to suggest that we expect that the 

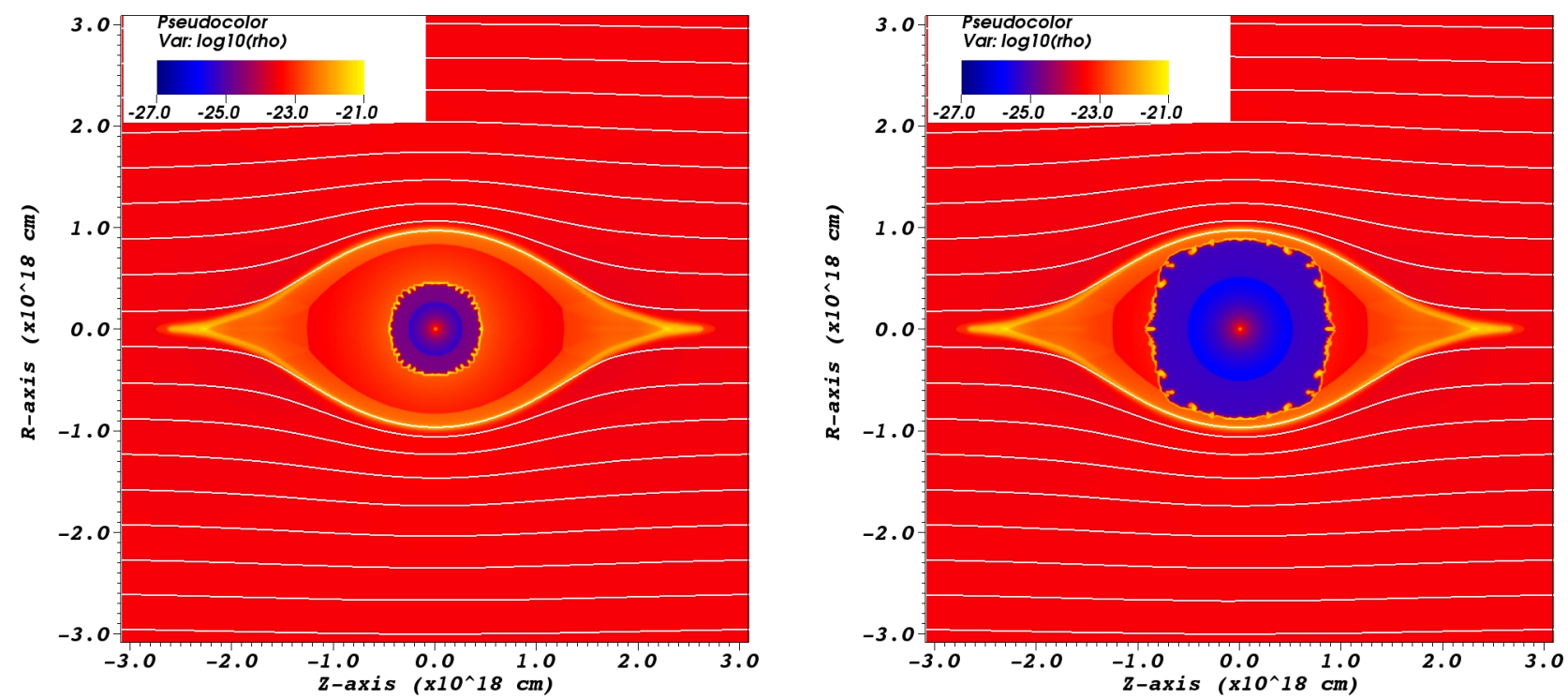

Fig. 15. Evolution of a PN in an eye-shaped AGB-wind bubble, showing the gas density [cgs.] and magnetic field lines at 2000 (left) and 4000 (right) years after the onset of the post-AGB phase. The PN, which starts out spherically (left), starts to feel the influence of the eye-like shape when it encounters the termination shock of the AGB wind (right).
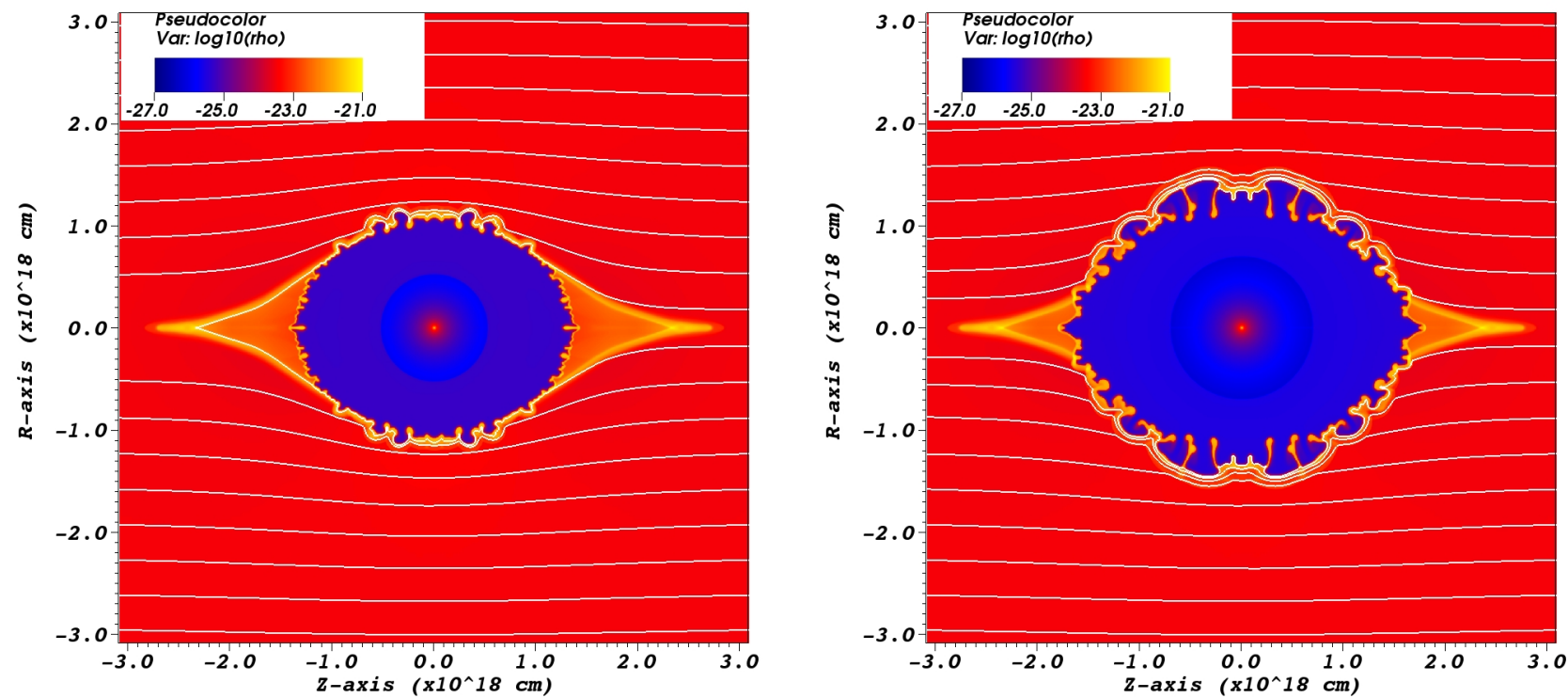

Fig. 16. Similar to Fig. 15 but 6000 (left) and 8000 years (right) after the transition from AGB to post-AGB. The PN becomes ovoid due to the asymmetry of the medium into which it expands. Note the dependence of the wavelength of the instabilities on the point at which the shell interacts with the magnetic field (right).

AGB phase would be as short as 0.1 Myr. Our sole purpose is to demonstrate what would happen if a PN were to form in an eye-shaped, AGB-wind bubble. We estimate the post-AGB wind to have a mass loss rate of $10^{-7} M_{\odot} \mathrm{yr}^{-1}$ and a terminal velocity of $1500 \mathrm{~km} \mathrm{~s}^{-1}$, which is reminiscent of the input parameters used by García-Segura et al. (1999). We do not consider the AGB-superwind (Tanabé et al. 1997; Kwok 2000, and reference therein). Although the effects of these additional factors are not trivial, they go beyond the scope of this paper.

\subsection{Results}

Figures 15 and 16 show the evolution of the PN at intervals of 2000 years. Initially, the PN is spherically symmetric (left panel of Fig. 15.) This is to be expected, because the interaction is between the spherically-symmetric post-AGB wind and the spherically-symmetric, free-streaming AGB wind. Because the swept-up shell is thin and highly compressed with thermal pressure from the shocked post-AGB wind on one side and rampressure from its own motion on the other, it is subject to linear thin-shell instabilities (Vishniac 1983). In addition, it shows sign of Rayleigh-Taylor instabilities with clumps of high density material moving from the shell into the low-density gas of the shocked post-AGB wind. If observed at this particular moment in time, the outer, eye-like shell may be visible as a halo around the PN. Such halos have been observed for several PNe, such as Abell 30 (Guerrero et al. 2012) and NGC 6751 (Clark et al. 2010), but are usually spherical in shape and can also be 

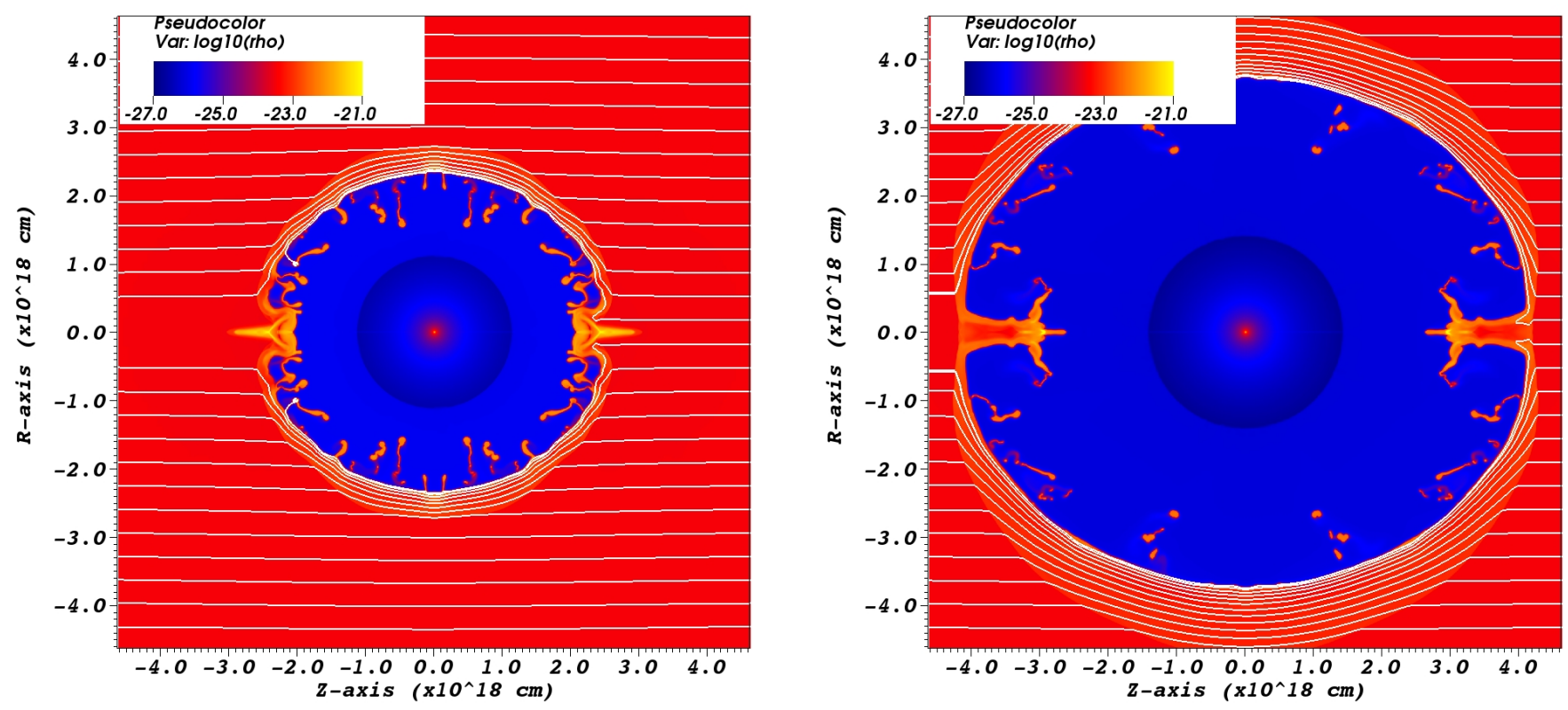

Fig. 17. Similar to Figs. 15, 16 but 15000 (left) and 30000 years (right) after the transition from AGB to post-AGB. The PN encounters the high density points of the eye, which slows down the expansion along the $z$-axis.

attributed to mass ejecta from a thermal pulse rather than the wind-ISM interaction. When the PN hits the termination shock of the the AGB wind bubble (right panel of Fig. 15), it slows down. This is partially because it has to overcome the thermal pressure of the shocked AGB wind, but primarily it now expands into a constant density medium rather than the $1 / r^{2}$ density profile of a free-streaming wind. As a result, the expansion velocity decreases over time, rather than remaining constant. Because the PN hits the termination shock first along the direction perpendicular to the magnetic field, the PN departs from being spherical symmetric and becomes ovoid (left panel of Fig. 16), a tendency that continues as it fills the AGB wind bubble, giving the PN an eye-like appearance, such as that observed for Abell 70 (Miszalski et al. 2012).

When the PN hits the outer shell of the AGB wind bubble, it starts to interact with the interstellar magnetic field. Initially, this means little for the expansion speed, which is driven by a relatively powerful wind. ( $R_{\mathrm{B}}$ for the post-AGB wind in a $10 \mu \mathrm{G}$ magnetic field is approximately $1 \mathrm{pc}$.) However, the nature of the instabilities in the shell changes. The Rayleigh-Taylor instabilities on the inner side of the shell grow (right panel of Fig. 16.) On the outer edge of the shell, the PN continues to display thin-shell instabilities. The wavelength of both thin-shell and Rayleigh-Taylor instabilities varies smoothly along the shell with the longest wavelength occurring where the expansion of the shell is perpendicular to the field lines. This phenomenon, which combines the influence of both field strength and direction on the wavelength of a perturbation was described analytically by, for example, Jun et al. (1995) and Breitschwerdt et al. (2000).

Eventually, the PN encounters the tips of the eye, while it expands along the $z$-axis (Fig. 17). This changes the morphology of the PN because sweeping up these high-density structures slows down the expansion. As a result, the PN takes on a new shape, which is reminiscent of a bi-polar nebula, as was also demonstrated, albeit for much stronger fields, by FalcetaGonçalves \& Monteiro (2014). However, one should keep in mind that the symmetry axis here is the $z$-axis. Therefore, the nebula would only appear bi-polar when observed from the side. In $3 \mathrm{D}$, the structure would actually be an ovoid that has been indented at the tips. Furthermore, the left and right panels of Fig. 17 show the PN after 15000 and 30000 years respectively. By this time the central star would almost certainly have become a white dwarf, rendering the PN invisible. The outer shell is no longer thin because the magnetic pressure from the compressed field counteracts the compression. This results in a disappearance of the instabilities. The general shape of the nebula strongly resembles the results of Tomisaka $(1990,1992)$ and Ferriere et al. (1991) for the expansion of superbubbles into an interstellar magnetic field.

\section{Conclusions}

Even a weak interstellar magnetic field can distort an AGB wind bubble into an eye-like shape. However, whether such a shape would actually be observed depends on other parameters, such as the stellar motion through the ISM and the visibility of the shell at the time that it has an eye-like shape. From our models we can conclude that visible eye-like nebulae are most likely to occur around stars that are subject to one or more of the following conditions:

1. Stars that are situated in a (relatively) strong interstellar magnetic field $\left(z^{5} \mu \mathrm{G}\right)$. This reduces the distance from the star at which the magnetic tension force starts to influence the shape of the shell.

2. Stars that are situated in a low density ISM $\left(\lesssim 2 / \mathrm{cm}^{3}\right)$. This reduces the influence of stellar motion and allows the magnetic field to dominate the restraining force that acts on the outside of the shell. Low density regions also tend to have a higher ISM temperature, which reduces the size of the nebula. This increases the density in the shell and the dust temperature, making the shell more observable.

3. Stars that have a relatively weak wind, allowing the magnetic field to dominate the force balance at an earlier stage. 
4. Stars that have jet spent only a relatively short time in the AGB phase. Once the star grows older, the eye-like structure disappears and the nebula grows larger, making it more difficult to observe.

5. Stars that have low proper motion (at least compared to the local sound speed). This gives the star more time to form an eye-like nebula before the stellar motion dominates the shape of the shell to form a bow shock.

In the direction perpendicular to the magnetic field, the AGB wind bubble remains small. Depending on mass-loss rate, magnetic field strength, and ISM density, the final short-axis radius of the bubble varies between 0.1 and $0.6 \mathrm{pc}$. This has potential implications for the evolution of $\mathrm{PNe}$, which may reach the outer edge of the AGB-wind bubble within their 10000 year lifespan.

Acknowledgements. We thank our anonymous referee for many insightful comments that helped us to improve our paper. A.J.v.M. acknowledges support from FWO, grant G.0277.08, KU Leuven GOA/2008/04 and GOA/2009/09. N.L.J.C. and L.D. acknowledge support from the Belgian Federal Science Policy Office via the PRODEX Programme of ESA. PACS has been developed by a consortium of institutes led by MPE (Germany) and including UVIE (Austria); KU Leuven, CSL, IMEC (Belgium); CEA, LAM (France); MPIA (Germany); INAF-IFSI/OAA/OAP/OAT, LENS, SISSA (Italy); IAC (Spain). This development has been supported by the funding agencies BMVIT (Austria), ESAPRODEX (Belgium), CEA/CNES (France), DLR (Germany), ASI/INAF (Italy), and CICYT/MCYT (Spain).

\section{Appendix A: Animations}

Movie A.1. Animation of simulation A1.

Movie A.2. Animation of simulation A2.

Movie A.3. Animation of simulation B1.

Movie A.4. Animation of simulation B2.

Movie A.5. Animation of simulation B3.

Movie A.6. Animation of simulation B4.

Movie A.7. Animation of simulation C1.

Movie A.8. Animation of simulation $\mathrm{C} 2$.

Movie A.9. Animation of simulation C3.

Movie A.10. Animation of simulation C4.

Movie A.11. Animation of simulation E1.

Movie A.12. Animation of planetary nebula.

\section{References}

Andersen, A. C. 2007, in Why Galaxies Care About AGB Stars, Their Importance as Actors and Probes, eds. F. Kerschbaum, C. Charbonnel, \& R. F. Wing, ASP Conf. Ser., 378, 170

Arndt, T. U., Fleischer, A. J., \& Sedlmayr, E. 1997, A\&A, 327, 614

Avedisova, V. S. 1972, Sov. Astron., 15, 708

Bergeat, J., \& Chevallier, L. 2005, A\&A, 429, 235

Binney, J., \& Merrifield, M. 1998, Galactic Astronomy (Princeton University Press)

Breitschwerdt, D., Freyberg, M. J., \& Egger, R. 2000, A\&A, 361, 303

Caselli, P., Walmsley, C. M., Terzieva, R., \& Herbst, E. 1998, ApJ, 499, 234

Clark, D. M., García-Díaz, M. T., López, J. A., Steffen, W. G., \& Richer, M. G. 2010, ApJ, 722, 1260
Cox, N. L. J., Kerschbaum, F., van Marle, A.-J., et al. 2012, A\&A, 537, A35

Dalgarno, A., \& McCray, R. A. 1972, ARA\&A, 10, 375

Decin, L., Cox, N. L. J., Royer, P., et al. 2012, A\&A, 548, A113

Elitzur, M., \& Ivezić, Ž. 2001, MNRAS, 327, 403

Falceta-Gonçalves, D., \& Monteiro, H. 2014, MNRAS, 438, 2853

Fatuzzo, M., Adams, F. C., \& Melia, F. 2006, ApJ, 653, L49

Ferriere, K. M., Mac Low, M.-M., \& Zweibel, E. G. 1991, ApJ, 375, 239

García-Segura, G., Langer, N., Różyczka, M., \& Franco, J. 1999, ApJ, 517, 767

Groenewegen, M. A. T., Waelkens, C., Barlow, M. J., et al. 2011, A\&A, 526, A162

Guerrero, M. A., Ruiz, N., Hamann, W.-R., et al. 2012, ApJ, 755, 129

Habing, H. J., \& Olofsson, H., eds. 2003, Asymptotic giant branch stars (Springer)

Han, J. L., Ferriere, K., \& Manchester, R. N. 2004, ApJ, 610, 820

Heiligman, G. M. 1980, MNRAS, 191, 761

Herwig, F. 2005, ARA\&A, 43, 435

Hoogzaad, S. N., Molster, F. J., Dominik, C., et al. 2002, A\&A, 389, 547

Jun, B.-I., Norman, M. L., \& Stone, J. M. 1995, ApJ, 453, 332

Kaastra, J. S., \& Mewe, R. 2000, in Atomic Data Needs for X-ray Astronomy, eds. M. A. Bautista, T. R. Kallman, \& A. K. Pradhan, 161

Keppens, R., Meliani, Z., van Marle, A. J., et al. 2012, J. Computt. Phys., 231, 718

Kerschbaum, F., Ladjal, D., Ottensamer, R., et al. 2010, A\&A, 518, L140

Kwok, S. 1975, ApJ, 198, 583

Kwok, S. 2000, The Origin and Evolution of Planetary Nebulae (Princeton University Press)

Kwok, S., Purton, C. R., \& Fitzgerald, P. M. 1978, ApJ, 219, L125

Lamers, H. J. G. L. M., \& Cassinelli, J. P. 1999, Introduction to Stellar Winds (Cambridge, UK: Cambridge University Press)

Maercker, M., Olofsson, H., Eriksson, K., Gustafsson, B., \& Schöier, F. L. 2010 A\&A, 511, A37

Marengo, M., Canil, G., Silvestro, G., et al. 1997, A\&A, 322, 924

Mayer, A., Jorissen, A., Kerschbaum, F., et al. 2013, A\&A, 549, A69

Minter, A. H., \& Spangler, S. R. 1996, ApJ, 458, 194

Miszalski, B., Boffin, H. M. J., Frew, D. J., et al. 2012, MNRAS, 419, 39

Pilbratt, G. L., Riedinger, J. R., Passvogel, T., et al. 2010, A\&A, 518, L1

Poglitsch, A., Waelkens, C., Geis, N., et al. 2010, A\&A, 518, L2

Powell, K. G., Roe, P. L., Linde, T. J., Gombosi, T. I., \& de Zeeuw, D. L. 1999, J. Comput. Phys., 154, 284

Ransom, R. R., Uyaniker, B., Kothes, R., \& Landecker, T. L. 2008, ApJ, 684, 1009

Schönrich, R., Binney, J., \& Dehnen, W. 2010, MNRAS, 403, 1829

Schure, K. M., Kosenko, D., Kaastra, J. S., Keppens, R., \& Vink, J. 2009, A\&A, 508,751

Shu, F. H. 1983, ApJ, 273, 202

Soker, N., \& Dgani, R. 1997, ApJ, 484, 277

Soker, N., \& Zucker, D. B. 1997, MNRAS, 289, 665

Stevens, I. R., Blondin, J. M., \& Pollock, A. M. T. 1992, ApJ, 386, 265

Tanabé, T., Nishida, S., Matsumoto, S., et al. 1997, Nature, 385, 509

Terzian, Y. 1997, in Planetary Nebulae, eds. H. J. Habing, \& H. J. G. L. M. Lamers, IAU Symp., 180, 29

Tielens, A. G. G. M. 2005, The Physics and Chemistry of the Interstellar Medium (Princeton University Press)

Tomisaka, K. 1990, ApJ, 361, L5

Tomisaka, K. 1992, PASJ, 44, 177

Tweedy, R. W., Martos, M. A., \& Noriega-Crespo, A. 1995, ApJ, 447, 257

van Marle, A. J., Meliani, Z., Keppens, R., \& Decin, L. 2011, ApJ, 734, L26

van Marle, A. J., Decin, L., \& Meliani, Z. 2014, A\&A, 561, A152

Villaver, E., García-Segura, G., \& Manchado, A. 2002, ApJ, 571, 880

Villaver, E., García-Segura, G., \& Manchado, A. 2003, ApJ, 585, L49

Villaver, E., Manchado, A., \& García-Segura, G. 2012, ApJ, 748, 94

Vishniac, E. T. 1983, ApJ, 274, 152

Wareing, C. J., Zijlstra, A. A., \& O'Brien, T. J. 2007, MNRAS, 382, 1233

Weaver, R., McCray, R., Castor, J., Shapiro, P., \& Moore, R. 1977, ApJ, 218, 377

Wilkin, F. P. 1996, ApJ, 459, L31

Woods, P. M., Walsh, C., Cordiner, M. A., \& Kemper, F. 2012, MNRAS, 426, 2689

Zhukovska, S., \& Henning, T. 2013, A\&A, 555, A99

Zhukovska, S., Gail, H.-P., \& Trieloff, M. 2008, A\&A, 479, 453 